ALEA, Lat. Am. J. Probab. Math. Stat. 16, 49-83 (2019)

\title{
The survival probability of the high-dimensional contact process with random vertex weights on the oriented lattice
}

\author{
Xiaofeng Xue \\ School of Science, Beijing Jiaotong University \\ No.3 Shangyuancun, Haidian District, \\ Beijing, China. \\ E-mail address: xf xue@bjtu.edu.cn \\ URL: http://faculty.bjtu.edu.cn/8975/
}

\begin{abstract}
This paper is a further investigation of the problem studied in Xue (2015). We are concerned with the contact process with random vertex weights on the oriented lattice. Our main result gives the asymptotic behavior of the survival probability of the process conditioned on only one vertex being infected at $t=0$ as the dimension grows to infinity. A SIR model and a branching process with random vertex weights are the main auxiliary tools for the proof of the main result.
\end{abstract}

\section{Introduction}

In this paper we are concerned with the contact process with random vertex weights on the oriented lattice $\mathbb{Z}_{+}^{d}$ for $d$ sufficiently large, where $\mathbb{Z}_{+}=\{0,1,2, \ldots\}$. This paper is a further investigation of the problem studied in Xue (2015), which deals with the critical value of the aforesaid process. First we introduce some notations and definitions. For $x=\left(x_{1}, \ldots, x_{d}\right) \in \mathbb{Z}_{+}^{d}$, we define

$$
\|x\|=\sum_{j=1}^{d} x_{j}
$$

as the $l_{1}$ norm of $x$. For $1 \leq j \leq d$, we use $e_{j}$ to denote the $j$ th elementary unit vector of $\mathbb{Z}_{+}^{d}$, i.e.,

$$
e_{j}=(0, \ldots, 0, \underset{j \mathrm{th}}{1}, 0, \ldots, 0)
$$

Received by the editors September 30th, 2017; accepted October 21th, 2018.

2010 Mathematics Subject Classification. 60K35.

Key words and phrases. Survival probability, contact process, oriented lattice.

Research supported by Beijing Jiaotong University and the National Natural Science Foundation of China. 
We use $O$ to denote the origin of $\mathbb{Z}_{+}^{d}$. For $x, y \in \mathbb{Z}_{+}^{d}$, we write $x \rightarrow y$ when and only when

for some $j \in\{1,2, \ldots, d\}$.

$$
y-x=e_{j}
$$

Let $\rho$ be a random variable such that $P(\rho \in[0, M])=1$ for some $M \in(0,+\infty)$ and $P(\rho>0)>0$, then we assign an independent copy $\rho(x)$ of $\rho$ on each vertex $x \in \mathbb{Z}_{+}^{d} . \rho(x)$ is called the vertex weight of $x$. We assume all these vertex weights are independent. After the vertex weights are given, the contact process $\left\{C_{t}\right\}_{t \geq 0}$ on $\mathbb{Z}_{+}^{d}$ with vertex weights $\{\rho(x)\}_{x \in \mathbb{Z}_{+}^{d}}$ is a continuous time Markov process with state space

$$
X=\left\{A: A \subseteq \mathbb{Z}_{+}^{d}\right\}
$$

and transition rates function given by

$$
C_{t} \rightarrow \begin{cases}C_{t} \backslash\{x\} & \text { at rate } 1 \text { if } x \in C_{t}, \\ C_{t} \bigcup\{x\} & \text { at rate } \frac{\lambda}{d} \sum_{y: y \rightarrow x} \rho(x) \rho(y) 1_{\left\{y \in C_{t}\right\}} \text { if } x \notin C_{t},\end{cases}
$$

where $\lambda$ is a positive constant called the infection rate while $1_{A}$ is the indicator function of the event $A$.

Intuitively, the process describes the spread of an epidemic on $\mathbb{Z}_{+}^{d}$. Vertices in $C_{t}$ are infected while vertices out of $C_{t}$ are healthy. An infected vertex waits for an exponential time with rate one to become healthy while a healthy vertex $x$ may be infected by an infected vertex $y$ when and only when $y \rightarrow x$. The infection occurs at a rate proportional to the product of the weights on these two vertices.

The (classic) contact process is introduced by Harris (1974), where $\rho \equiv 1$ and infection occurs between nearest (un-oriented) neighbors. For a detailed survey of the classic contact process, see Chapter 6 of Liggett (1985) and Part 1 of Liggett (1999).

The contact process with random vertex weights is first introduced in Peterson (2011) on the complete graph $K_{n}$ by Peterson, where a phase transition consistent with the mean-field analysis is shown. In detail, the infection dies out in $O(\log n)$ units of time with high probability when $\lambda<\frac{1}{E\left(\rho^{2}\right)}$ or survives for $\exp \{O(n)\}$ units of time with high probability when $\lambda>\frac{1}{E\left(\rho^{2}\right)}$. In Xue (2015), Xue studies this process on the oriented lattice and gives the asymptotic behavior of the critical value of the process as the dimension $d$ grows to infinity. When $P(\rho=1)=p=$ $1-P(\rho=0)$ for some $p \in(0,1)$, the model reduces to the contact process on clusters of the site percolation, which is a special case of the model introduced in Bertacchi et al. (2011) with $n=1$. In Bertacchi et al. (2011), Bertacchi, Lanchier and Zucca study the contact process on $G \times K_{n}$, where $G$ is the infinite open cluster of the site percolation while $K_{n}$ is the complete graph with $n$ vertices. Criteria judging whether the process survives are given.

If the i.i.d. weights are assigned on the edges instead of on the vertices, the model turns into the contact process with random edge weights, which is first introduced by Yao and Chen (2012), where a complete convergence theorem is shown.

\section{Main results}

In this section we give our main results. First we introduce some notations and definitions. We assume that $\{\rho(x)\}_{x \in \mathbb{Z}_{+}^{d}}$ are defined under the probability 
space $\left(\Omega_{d}, \mathcal{F}_{d}, \mu_{d}\right)$. The expectation with respect to $\mu_{d}$ is denoted by $E_{\mu_{d}}$. For $\omega \in \Omega_{d}$, we denote by $P_{\lambda, \omega}$ the probability measure of our model with vertex weights $\{\rho(x, \omega)\}_{x \in \mathbb{Z}_{+}^{d}}$. $P_{\lambda, \omega}$ is called the quenched measure. The expectation with respect to $P_{\lambda, \omega}$ is denoted by $E_{\lambda, \omega}$. We define

$$
P_{\lambda, d}(\cdot)=E_{\mu_{d}}\left[P_{\lambda, \omega}(\cdot)\right]=\int P_{\lambda, \omega}(\cdot) \mu_{d}(d \omega),
$$

which is called the annealed measure. The expectation with respect to $P_{\lambda, d}$ is denoted by $E_{\lambda, d}$.

For any $A \subseteq \mathbb{Z}_{+}^{d}$, we write $C_{t}$ as $C_{t}^{A}$ when $C_{0}=A$. If $A=\{x\}$ for some $x \in \mathbb{Z}_{+}^{d}$, we write $C_{t}^{A}$ as $C_{t}^{x}$ instead of $C_{t}^{\{x\}}$.

For $\lambda>\frac{1}{E\left(\rho^{2}\right)}$, where $E$ is the expectation with respect to $\rho$, there is a unique solution $\theta>0$ to the equation

$$
E\left(\frac{\lambda \rho^{2}}{1+\lambda \rho \theta}\right)=1
$$

Now we give the main result of this paper.

Theorem 2.1. For any $\lambda>\frac{1}{E\left(\rho^{2}\right)}$ and $\theta$ defined as in Equation (2.1),

$$
\lim _{d \rightarrow+\infty} P_{\lambda, d}\left(C_{t}^{O} \neq \emptyset, \forall t \geq 0\right)=E\left(\frac{\lambda \rho \theta}{1+\lambda \rho \theta}\right) .
$$

Theorem 2.1 gives the asymptotic behavior of the survival probability of the process conditioned on $O$ being the unique initially infected vertex as the dimension $d$ grows to infinity. The theorem only deals with the case where $\lambda>\frac{1}{E\left(\rho^{2}\right)}$ because

$$
\lim _{d \rightarrow+\infty} P_{\lambda, d}\left(C_{t}^{O} \neq \emptyset, \forall t \geq 0\right)=0
$$

for any $\lambda<\frac{1}{E\left(\rho^{2}\right)}$ according to the main theorem given in Xue (2015), which shows that the critical value of the infection rate of the model converges to $\frac{1}{E\left(\rho^{2}\right)}$ as $d \rightarrow+\infty$.

When $\rho \equiv 1$, we have the following direct corollary.

Corollary 2.2. If $\rho \equiv 1$ and $\lambda>1$, then

$$
\lim _{d \rightarrow+\infty} P_{\lambda, d}\left(C_{t}^{O} \neq \emptyset, \forall t \geq 0\right)=\frac{\lambda-1}{\lambda} .
$$

The counterpart of Corollary 2.2 for the classic contact process on the lattice is given in Schonmann and Vares (1986). An independent proof for the same result is given in Xue (2017a), the author of which was unware of reference Schonmann and Vares (1986).

The counterpart of Theorem 2.1 for the contact process with random edges weights on the (un-oriented) lattice is given in Xue (2017b). It is claimed in Xue (2017b) that

$$
\lim _{d \rightarrow+\infty} P_{\lambda, d}\left(C_{t}^{O} \neq \emptyset, \forall t \geq 0\right)=\frac{\lambda E \rho-1}{\lambda E \rho}
$$

for the process with edge weights which are independent copies of $\rho$ and infection rate $\lambda>\frac{1}{E \rho}$.

As an auxiliary tool for the proof of Theorem 2.1, we introduce a SIR (susceptibleinfected-recovered) model with random vertex weights on $\mathbb{Z}_{+}^{d}$. 
After the vertex weights $\{\rho(x)\}_{x \in \mathbb{Z}_{+}^{d}}$ are given, the SIR model $\left\{\left(S_{t}, I_{t}\right)\right\}_{t \geq 0}$ is a continuous-time Markov process with state space

$$
X_{2}=\left\{(S, I): S, I \subseteq \mathbb{Z}_{+}^{d}, S \bigcap I=\emptyset\right\}
$$

and transition rates function given by

$$
\left(S_{t}, I_{t}\right) \rightarrow \begin{cases}\left(S_{t}, I_{t} \backslash\{x\}\right) & \text { at rate } 1 \text { if } x \in I_{t}, \\ \left(S_{t} \backslash\{x\}, I_{t} \bigcup\{x\}\right) & \text { at rate } \frac{\lambda}{d} \sum_{y: y \rightarrow x} \rho(x) \rho(y) 1_{\left\{y \in I_{t}\right\}} \text { if } x \in S_{t} .\end{cases}
$$

For the SIR model, an infected vertex waits for an exponential time with rate one to become recovered while a recovered vertex can never be infected again.

We write $\left(S_{t}, I_{t}\right)$ as $\left(S_{t}^{A}, I_{t}^{A}\right)$ when $\left(S_{0}, I_{0}\right)=\left(\mathbb{Z}_{+}^{d} \backslash A, A\right)$, then it is easy to check that

$$
P_{\lambda, d}\left(I_{t}^{O} \neq \emptyset, \forall t \geq 0\right) \leq P_{\lambda, d}\left(C_{t}^{O} \neq \emptyset, \forall t \geq 0\right) .
$$

One way to check this inequality is to utilize the basic coupling of Markov processes (see Section 3.1 of Liggett (1985)), we omit the details. As a result, to prove Theorem 2.1, we only need to show that

$$
\liminf _{d \rightarrow+\infty} P_{\lambda, d}\left(I_{t}^{O} \neq \emptyset, \forall t \geq 0\right) \geq E\left(\frac{\lambda \rho \theta}{1+\lambda \rho \theta}\right)
$$

and

$$
\limsup _{d \rightarrow+\infty} P_{\lambda, d}\left(C_{t}^{O} \neq \emptyset, \forall t \geq 0\right) \leq E\left(\frac{\lambda \rho \theta}{1+\lambda \rho \theta}\right) .
$$

The proof of Theorem 2.1 is divided into three sections. In Section 3, we introduce a branching process $\left\{W_{n}\right\}_{n \geq 0}$ with random vertex weights on the oriented rooted tree $\mathbb{T}^{d}$. We will show that the probability that the branching process survives converges to $E\left(\frac{\lambda \rho \theta}{1+\lambda \rho \theta}\right)$ as $d \rightarrow+\infty$.

In Section 4, we give the proof of Equation (2.3). The proof relies on a coupling relationship between the branching process and the SIR model. A technique introduced in Xue (2015) is utilized.

In Section 5, we give the proof of Equation (2.4). The proof relies on a coupling relationship between the three aforesaid processes.

\section{A branching process with vertex weights}

In this section we introduce a branching process with random vertex weights on the oriented rooted tree. We denote by $\mathbb{T}^{d}$ the rooted tree that the root has $d$ neighbors while any other vertex on the tree has $d+1$ neighbors. We denote by $\Upsilon$ the root of the tree. There is a function $f: \mathbb{T}^{d} \rightarrow\{0,1,2, \ldots\}$ which satisfies the following conditions.

(1) $f(\Upsilon)=0$.

(2) $f(x)=1$ for each neighbor $x$ of $\Upsilon$.

(3) For any $y \neq \Upsilon$, there is one neighbor $u$ of $y$ such that $f(u)=f(y)-1$ while there are $d$ neighbors $v$ of $y$ such that $f(v)=f(y)+1$.

For $x, y \in \mathbb{T}^{d}$, we write $x \Rightarrow y$ when and only when $x$ and $y$ are neighbors and $f(y)=f(x)+1$.

Intuitively, $\Upsilon$ is the ancestor of a family and has $d$ sons. Each other individual in this family has one father and $d$ sons. $x \Rightarrow y$ when and only when $y$ is a son of $x$. 
We assume that $\{\rho(x)\}_{x \in \mathbb{T}^{d}}$ are i.i.d. copies of the random variable $\rho$, which is defined as in Section 1. After the vertex weights are given, we assume that $Y(x)$ is an exponential time with rate one for each $x \in \mathbb{T}^{d}$ while $U(x, y)$ is an exponential time with rate $\frac{\lambda}{d} \rho(x) \rho(y)$ for any $x, y \in \mathbb{T}^{d}$ such that $x \Rightarrow y$. We assume that all these exponential times are independent under the given vertex weights. Then, the branching process $\left\{W_{n}\right\}_{n \geq 0}$ is defined as follows.

(1) $W_{0}=\Upsilon$.

(2) For $n \geq 0, W_{n+1}=\left\{y: x \Rightarrow y\right.$ and $U(x, y)<Y(x)$ for some $\left.x \in W_{n}\right\}$.

$\left\{W_{n}\right\}_{n \geq 0}$ describes the spread of a SIR epidemic on $\mathbb{T}^{d}$. Initially, $\Upsilon$ is infected. A healthy vertex may only be infected by its father. If $x$ is infected, then $x$ waits for an exponential time with rate one to become recovered while waits for an exponential time with rate $\frac{\lambda}{d} \rho(x) \rho(y)$ to infect the son $y$. The infection really occurs when and only when $y$ is infected before the moment when $x$ is recovered, i.e., $U(x, y)<Y(x)$.

Similar with what we have done in Section 2, we denote by $\widehat{P}_{\lambda, \omega}$ the quenched measure of the branching process with respect to the random environment $\omega$ in the space where $\{\rho(x)\}_{x \in \mathbb{T}^{d}}$ are defined. We denote by $\widehat{P}_{\lambda, d}$ the annealed measure. Note that according to our definition, for $x \Rightarrow y \Rightarrow z, U(x, y)$ and $U(y, z)$ are independent under $\widehat{P}_{\lambda, \omega}$ while positively correlated under $\widehat{P}_{\lambda, d}$.

The branching process $\left\{W_{n}\right\}_{n \geq 0}$ with random vertex weights on the oriented tree $\mathbb{T}^{d}$ is first introduced in Pan et al. (2017). Some results obtained in Pan et al. (2017) will be directly utilized in this section.

The following lemma is crucial for us to prove Theorem 2.1.

Lemma 3.1. For any $\lambda>\frac{1}{E\left(\rho^{2}\right)}$ and $\theta$ defined as in Equation (2.1),

$$
\lim _{d \rightarrow+\infty} \widehat{P}_{\lambda, d}\left(W_{n} \neq \emptyset, \forall n \geq 0\right)=E\left(\frac{\lambda \rho \theta}{1+\lambda \rho \theta}\right) .
$$

The remainder of this section is devoted to the proof of Lemma 3.1. From now on we assume that $\lambda>\frac{1}{E\left(\rho^{2}\right)}$. Let $M$ be defined as in Section 1 . For any $s \in[0, M]$, we define

$$
F_{d}(s)=\widehat{P}_{\lambda, d}\left(W_{n}=\emptyset \text { for some } n \geq 0 \mid \rho(\Upsilon)=s\right),
$$

then the following two lemmas are crucial for us to prove Lemma 3.1.

Lemma 3.2. If $\left\{d_{l}\right\}_{l \geq 1}$ is a subsequence of $1,2,3, \ldots$ such that

$$
\lim _{l \rightarrow+\infty} F_{d_{l}}(s)
$$

exists for any $s \in[0, M]$, then

$$
\lim _{l \rightarrow+\infty} F_{d_{l}}(s)=\frac{1}{1+\lambda s \theta}
$$

for any $s \in[0, M]$.

Lemma 3.3. For $d \geq 1$ and $0 \leq s<t \leq M$,

$$
\left|F_{d}(s)-F_{d}(t)\right| \leq \lambda(t-s) M .
$$

We first show how to utilize Lemmas 3.2 and 3.3 to prove Lemma 3.1. The proofs of Lemmas 3.2 and 3.3 are given at the end of this section. 
Proof of Lemma 3.1: If Lemma 3.1 does not hold, then there are a constant $\epsilon_{0}>0$ and a subsequence $\left\{a_{l}\right\}_{l \geq 1}$ of $1,2,3, \ldots$ such that

$$
\left|E\left(F_{a_{l}}(\rho)\right)-E\left(\frac{1}{1+\lambda \rho \theta}\right)\right|>\epsilon_{0},
$$

since

$$
\widehat{P}_{\lambda, d}\left(W_{n} \neq \emptyset, \forall n \geq 0\right)=1-E\left(F_{d}(\rho)\right) .
$$

Since $0 \leq F_{d}(\cdot) \leq 1$, according to a classic procedure of picking subsequences, there is a subsequence $\left\{d_{j}\right\}_{j \geq 1}$ of $\left\{a_{l}\right\}_{l \geq 1}$ such that

$$
\lim _{j \rightarrow+\infty} F_{d_{j}}(r)
$$

exists for any $r \in \mathbb{Q}$. We use $F_{\Delta}(r)$ to denote $\lim _{j \rightarrow+\infty} F_{d_{j}}(r)$. It is obvious that $F_{d}(s)$ is decreasing with $s$ for each $d \geq 1$, then

$$
F_{d}\left(r_{1}\right) \geq F_{d}(s) \geq F_{d}\left(r_{2}\right) \text { and } F_{\Delta}\left(r_{1}\right) \geq F_{\Delta}\left(r_{2}\right)
$$

for any $r_{1}<s<r_{2}, r_{1}, r_{2} \in \mathbb{Q}$. As a result, it is reasonable to define

$$
F_{\Delta}^{-}(s)=\lim _{r \uparrow s, r \in \mathbb{Q}} F_{\Delta}(r) \text { and } F_{\Delta}^{+}(s)=\lim _{r \downarrow s, r \in \mathbb{Q}} F_{\Delta}(r)
$$

for any $s \notin \mathbb{Q}$ and hence

$$
\limsup _{j \rightarrow+\infty} F_{d_{j}}(s) \leq F_{\Delta}^{-}(s) \text { while } \liminf _{j \rightarrow+\infty} F_{d_{j}}(s) \geq F_{\Delta}^{-}(s) .
$$

By Lemma 3.3,

$$
\left|F_{\Delta}\left(r_{1}\right)-F_{\Delta}\left(r_{2}\right)\right| \leq \lambda M\left(r_{2}-r_{1}\right)
$$

for $r_{1}<s<r_{2}, r_{1}, r_{2} \in \mathbb{Q}$. Therefore, let $r_{1} \uparrow s$ and $r_{2} \downarrow s$,

$$
F_{\Delta}^{-}(s)=F_{\Delta}^{+}(s)
$$

and

$$
\lim _{j \rightarrow+\infty} F_{d_{j}}(s)=F_{\Delta}^{-}(s)=F_{\Delta}^{+}(s)
$$

for any $s \notin \mathbb{Q}$. For $s \notin \mathbb{Q}$, we use $F_{\Delta}(s)$ to denote $F_{\Delta}^{-}(s)$, which equals $F_{\Delta}^{+}(s)$. As a result,

$$
\lim _{j \rightarrow+\infty} F_{d_{j}}(s)
$$

exists for any $s \in[0, M]$ and

$$
\lim _{j \rightarrow+\infty} F_{d_{j}}(s)=F_{\Delta}(s)
$$

for any $s \in[0, M]$. Then, by Lemma 3.2,

$$
F_{\Delta}(s)=\frac{1}{1+\lambda s \theta}
$$

for any $s \in[0, M]$ and hence

$$
\lim _{j \rightarrow+\infty} E\left(F_{d_{j}}(\rho)\right)=E\left(\frac{1}{1+\lambda \rho \theta}\right) .
$$

However, this is contradictory with Equation (3.1) since $\left\{d_{j}\right\}_{j \geq 1}$ is a subsequence of $\left\{a_{l}\right\}_{l \geq 1}$. As a result, Lemma 3.1 holds and the proof is complete.

At last we give the proof of Lemmas 3.2 and 3.3 . 
Proof of Lemma 3.2: For $\Upsilon \Rightarrow y$, conditioned on $Y(\Upsilon), \rho(\Upsilon), \rho(y)$, the probability that $\Upsilon$ infects $y$ is

$$
\begin{aligned}
& \widehat{P}_{\lambda, d}(U(\Upsilon, y)<Y(\Upsilon) \mid \rho(\Upsilon), Y(\Upsilon), \rho(y)) \\
& =1-e^{-\frac{\lambda}{d} \rho(\Upsilon) \rho(y) Y(\Upsilon)}
\end{aligned}
$$

If $\left\{W_{n}\right\}_{n \geq 0}$ dies out, then for any $y$ such that $\Upsilon$ infects $y$, the epidemic on the subtree consisted of $y$ and its descendant must die out, the probability of which is $F_{d}(\rho(y))$. As a result,

$$
\begin{aligned}
& \widehat{E}_{\lambda, d}\left[W_{n}=\emptyset \text { for some } n \geq 0 \mid \rho(\Upsilon), Y(\Upsilon),\{\rho(y): \Upsilon \Rightarrow y\}\right] \\
& =\prod_{y: \Upsilon \Rightarrow y}\left(F_{d}(\rho(y))\left(1-e^{-\frac{\lambda}{d} \rho(\Upsilon) \rho(y) Y(\Upsilon)}\right)+e^{-\frac{\lambda}{d} \rho(\Upsilon) \rho(y) Y(\Upsilon)}\right)
\end{aligned}
$$

and hence

$$
F_{d}(s)=\widehat{E}_{\lambda, d}\left[\prod_{y: \Upsilon \Rightarrow y}\left(F_{d}(\rho(y))\left(1-e^{-\frac{\lambda}{d} s \rho(y) Y(\Upsilon)}\right)+e^{-\frac{\lambda}{d} s \rho(y) Y(\Upsilon)}\right)\right] .
$$

Since $\{\rho(y): \Upsilon \Rightarrow y\}$ are independent,

$$
F_{d}(s)=\widehat{E}_{\lambda, d}\left[\left(H_{d}(Y(\Upsilon))\right)^{d}\right]=E\left[\left(H_{d}\left(Y_{0}\right)\right)^{d}\right]
$$

where

$$
H_{d}(t)=E\left(F_{d}(\rho)\left(1-e^{-\frac{\lambda}{d} s t \rho}\right)+e^{-\frac{\lambda}{d} s t \rho}\right)
$$

for any $t \geq 0$ and $Y_{0}$ is an exponential time with rate one defined under some space we do not care. For $s \in[0, M]$, we use $F(s)$ to denote $\lim _{l \rightarrow+\infty} F_{d_{l}}(s)$, which exists according the assumption of Lemma 3.2. Then,

$$
\lim _{l \rightarrow+\infty} d_{l}\left[1-F_{d_{l}}(\rho)\right]\left[1-e^{-\frac{\lambda}{d_{l}} s t \rho}\right]=\lambda s t \rho(1-F(\rho))
$$

while

$$
d\left[1-F_{d}(\rho)\right]\left[1-e^{-\frac{\lambda}{d} s t \rho}\right] \leq 2 \lambda s t \rho \leq 2 \lambda s t M
$$

for any $d \geq 1$. Hence, according to the dominated convergence theorem,

$$
\lim _{l \rightarrow+\infty} d_{l}\left(H_{d_{l}}(t)-1\right)=-\lambda s t E(\rho(1-F(\rho))) .
$$

According to the theory of calculus, if $a_{d} \rightarrow 0, c_{d} \rightarrow+\infty$ and $a_{d} c_{d} \rightarrow c$, then $\left(1+a_{d}\right)^{c_{d}} \rightarrow e^{c}$. Therefore, by Equation (3.3),

$$
\lim _{l \rightarrow+\infty}\left(H_{d_{l}}\left(Y_{0}\right)\right)^{d_{l}}=e^{-\lambda s Y_{0} E(\rho(1-F(\rho)))} .
$$

For each $d \geq 1$,

$$
\left(H_{d}\left(Y_{0}\right)\right)^{d} \leq\left(1-e^{-\frac{\lambda}{d} s t \rho}+e^{-\frac{\lambda}{d} s t \rho}\right)^{d}=1 .
$$

Then, according to the dominated convergence theorem,

$$
\lim _{l \rightarrow+\infty} F_{d_{l}}(s)=\lim _{l \rightarrow+\infty} E\left[\left(H_{d_{l}}\left(Y_{0}\right)\right)^{d_{l}}\right]=E e^{-\lambda s Y_{0} \widetilde{\theta}}=\frac{1}{1+\lambda s \widetilde{\theta}}
$$


where $\widetilde{\theta}=E(\rho(1-F(\rho)))$. As a result, $F(s)=\frac{1}{1+\lambda s \widetilde{\theta}}$ for any $s$ and we only need to show that $\widetilde{\theta}=\theta$. According to the definition of $\widetilde{\theta}$,

$$
\widetilde{\theta}=E(\rho(1-F(\rho)))=E\left(\rho\left(1-\frac{1}{1+\lambda \rho \widetilde{\theta}}\right)\right)=E\left(\frac{\lambda \rho^{2} \widetilde{\theta}}{1+\lambda \rho \widetilde{\theta}}\right)
$$

Therefore, to prove $\theta=\widetilde{\theta}$ we only need to show that $\tilde{\theta} \neq 0$. This fact follows directly from the fact that

$$
\limsup _{d \rightarrow+\infty} E\left(F_{d}(\rho)\right)<1
$$

when $\lambda>\frac{1}{E\left(\rho^{2}\right)}$, which is proved in Pan et al. (2017).

Proof of Lemma 3.3: We denote by $\left\{W_{n}^{s}\right\}_{n \geq 0}$ the branching process conditioned on $\rho(\Upsilon)=s$ and denote by $\left\{W_{n}^{t}\right\}_{n \geq 0}$ the branching process conditioned on $\rho(\Upsilon)=t$. We couple these two branching processes in a same probability space as follows. For any $x \in \mathbb{T}^{d}$, we assume that these two processes utilize the same exponential time $Y(x)$ with rate one. For any $x \neq \Upsilon$ and $x \Rightarrow z$, we assume that these two processes utilize the same exponential time $U(x, z)$ with rate $\frac{\lambda}{d} \rho(x) \rho(z)$. For each $y$ that $\Upsilon \Rightarrow y$, we assume that $\left\{W_{n}^{s}\right\}_{s \geq 0}$ utilizes an exponential time $U_{s}(\Upsilon, y)$ with rate $\frac{\lambda}{d} s \rho(y)$ while $\left\{W_{n}^{t}\right\}_{t \geq 0}$ utilizes an exponential time

$$
U_{t}(\Upsilon, y)=\inf \left\{U_{s}(\Upsilon, y), U_{t-s}(\Upsilon, y)\right\},
$$

where $U_{t-s}(\Upsilon, y)$ is an exponential time with rate $\frac{\lambda}{d}(t-s) \rho(y)$ and is independent of $U_{s}(\Upsilon, y), Y(\Upsilon)$ under the quenched measure. Therefore, $U_{t}(\Upsilon, y)$ is an exponential time with rate $\frac{\lambda}{d} t \rho(y)$. According to the coupling of $\left\{W_{n}^{s}\right\}_{n \geq 0}$ and $\left\{W_{n}^{t}\right\}_{n \geq 0}$,

$$
\begin{aligned}
\left|F_{d}(t)-F_{d}(s)\right| & =\widehat{P}_{\lambda, d}\left(\left\{W_{n}^{t}\right\}_{n \geq 0} \text { survives while }\left\{W_{n}^{s}\right\}_{n \geq 0} \text { dies out }\right) \\
& \leq \widehat{P}_{\lambda, d}\left(W_{1}^{s} \neq W_{1}^{t}\right) \\
& =\widehat{P}_{\lambda, d}\left(U_{t-s}(\Upsilon, y)<Y(\Upsilon)<U_{s}(\Upsilon, y) \text { for some } y\right) \\
& \leq \sum_{y: \Upsilon \Rightarrow y} \widehat{P}_{\lambda, d}\left(U_{t-s}(\Upsilon, y)<Y(\Upsilon)<U_{s}(\Upsilon, y)\right) \\
& =\sum_{y: \Upsilon \Rightarrow y} \widehat{E}_{\lambda, d}\left[e^{-\frac{\lambda}{d} s \rho(y) Y(\Upsilon)}-e^{-\frac{\lambda}{d} t \rho(y) Y(\Upsilon)}\right] \\
& =\sum_{y: \Upsilon \Rightarrow y} \widehat{E}_{\lambda, d}\left[\frac{1}{1+\frac{\lambda}{d} s \rho(y)}-\frac{1}{1+\frac{\lambda}{d} t \rho(y)}\right] \\
& =d E\left[\frac{\frac{\lambda}{d}(t-s) \rho}{\left(1+\frac{\lambda s \rho}{d}\right)\left(1+\frac{\lambda t \rho}{d}\right)}\right] \leq d \frac{\lambda}{d}(t-s) M=\lambda(t-s) M
\end{aligned}
$$

and the proof is complete.

\section{Proof of Equation (2.3)}

In this section we give the proof of Equation (2.3). Throughout this section we assume that

$$
\lambda>\frac{1}{E\left(\rho^{2}\right)} \geq \frac{1}{M^{2}}
$$


where $M$ is defined as in Section 1. For later use, we assume that there exists $\epsilon>0$ that

$$
P(\rho=0 \text { or } \rho \in[\epsilon, M])=1 .
$$

This assumption is without loss of generality according to the following analysis. For $\rho$ not satisfying (4.1), we let $\rho_{m}=\rho 1_{\{\rho \geq 1 / m\}}$, then $\rho \geq \rho_{m}$ and $\rho_{m} \rightarrow \rho$ as $m \rightarrow+\infty$. It is obvious that $\rho_{m}$ satisfies (4.1) while the process with weights given by $\rho$ has larger probability to survive than that with weights given by $\rho_{m}$. As a result, if Equation (2.3) holds under assumption (4.1), then

$\liminf _{d \rightarrow+\infty} P_{\lambda, d, \rho}\left(I_{t}^{O} \neq \emptyset, \forall t \geq 0\right) \geq \liminf _{d \rightarrow+\infty} P_{\lambda, d, \rho_{m}}\left(I_{t}^{O} \neq \emptyset, \forall t \geq 0\right) \geq E\left(\frac{\lambda \rho_{m} \theta_{m}}{1+\lambda \rho_{m} \theta_{m}}\right)$

for any sufficiently large $m$, where $P_{\lambda, d, \rho}$ is the annealed measure of the process with vertex weights which are i.i.d copies of $\rho$ while $\theta_{m}$ satisfies

$$
E\left(\frac{\lambda \rho_{m}^{2}}{1+\lambda \rho_{m} \theta_{m}}\right)=1
$$

and it is easy to check that $\lim _{m \rightarrow+\infty} \theta_{m}=\theta$. Let $m \rightarrow+\infty$, then Equation (2.3) holds for general $\rho$.

First we give a sketch of the proof, which is inspired by the approach introduced in Xue (2017b). We divide $\mathbb{Z}_{+}^{d}$ into two parts $\Gamma_{1}$ and $\Gamma_{2}$ such that

$$
\Gamma_{1} \bigcap \Gamma_{2}=\left\{x:\|x\|=\left\lfloor\sigma_{0} \log d\right\rfloor\right\}
$$

where $\sigma_{0}$ is a positive constant. The first step is to show that with probability at least $E\left(\frac{\lambda \rho \theta}{1+\lambda \rho \theta}\right)+o(1)$ there exists $O\left(\frac{\sqrt{\log d}}{\log (\log d)}\right)$ vertices on $\Gamma_{1} \bigcap \Gamma_{2}$ which have been infected by $O$ through paths on $\Gamma_{1}$. The second step is to show that conditioned on $O\left(\frac{\sqrt{\log d}}{\log (\log d)}\right)$ vertices being initially infected on $\Gamma_{1} \cap \Gamma_{2}$, the SIR model on $\Gamma_{2}$ survives with high probability. To prove the first step, we construct a coupling between the SIR on $\mathbb{Z}_{+}^{d}$ and the branching process introduced in Section 3.

To give our proof, we introduce some definitions and notations. For sufficiently large $d$, we define $N(d)=\log (\log d), j(d)=d-\left\lfloor\frac{d}{N(d)}\right\rfloor+1$. Let $\sigma_{0}$ be a fixed constant such that

then we define

$$
\sigma_{0} \in\left(0, \frac{1}{10 \log \left(\lambda M^{2}\right)}\right),
$$

$$
\begin{aligned}
& \Gamma_{1}=\left\{x=\left(x_{1}, x_{2}, \ldots, x_{d}\right) \in \mathbb{Z}_{+}^{d}:\|x\| \leq\left\lfloor\sigma_{0} \log d\right\rfloor\right\}, \\
& \Gamma_{2}=\left\{x \in \mathbb{Z}_{+}^{d}:\|x\| \geq\left\lfloor\sigma_{0} \log d\right\rfloor\right\} .
\end{aligned}
$$

For $n \geq 0$, we define

$$
V_{n}=\left\{x \in \mathbb{Z}_{+}^{d}:\|x\|=n \text { and } x \in I_{t}^{O} \text { for some } t \geq 0\right\}
$$

as the set of vertices which have ever been infected with $l_{1}$ norm $n$. Since in the SIR model, infection can not occur repeatedly between neighbors, $\left\{V_{n}\right\}_{n \geq 0}$ can be defined equivalently as the following way. For each $x \in \mathbb{Z}_{+}^{d}$, let $\widetilde{Y}(x)$ be an exponential time with rate one. For any $x, y$ that $x \rightarrow y$, let $\widetilde{U}(x, y)$ be an exponential time with rate $\frac{\lambda}{d} \rho(x) \rho(y)$. We assume that all these exponential times are independent under the quenched measure with respect to the given edge weights, then 
(1) $V_{0}=\{O\}$.

(2) For each $n \geq 0$,

$$
V_{n+1}=\left\{y \in \mathbb{Z}_{+}^{d}: x \rightarrow y \text { and } \widetilde{U}(x, y)<\widetilde{Y}(x) \text { for some } x \in V_{n}\right\} .
$$

The intuitive explanation of the above definition is similar with that of the branching process introduced in Section 3. $\widetilde{Y}(x)$ is time $x$ waits for to become recovered after $x$ is infected while $\widetilde{U}(x, y)$ is the time $x$ waits for to infect $y$.

Let

$$
b_{0}=\frac{\lambda \epsilon \theta}{2(1+\lambda \epsilon \theta)} \text { and } K(d)=\left\lfloor\frac{b_{0} \sqrt{\log d}}{N(d)}\right\rfloor,
$$

then we have the following lemma.

\section{Lemma 4.1.}

$$
\liminf _{d \rightarrow+\infty} P_{\lambda, d}\left(\left|V_{\left\lfloor\sigma_{0} \log d\right\rfloor}\right| \geq K(d)\right) \geq E\left(\frac{\lambda \rho \theta}{1+\lambda \rho \theta}\right),
$$

where $\left|V_{\left\lfloor\sigma_{0} \log d\right\rfloor}\right|$ is the cardinality of $V_{\left\lfloor\sigma_{0} \log d\right\rfloor}$.

The proof of Lemma 4.1 is given in Subsection 4.2. As a preparation of this proof, we give a coupling of $\left\{W_{n}\right\}_{n \geq 0}$ and $\left\{V_{n}\right\}_{n \geq 0}$ in Subsection 4.1.

To execute the second step as we have introduced, we define

$$
\begin{gathered}
m(d)=\inf \left\{P_{\lambda, d}\left(I_{t}^{A} \neq \emptyset \text { for all } t \geq 0 \mid \rho(x)=\epsilon \text { for all } x \in A\right):\right. \\
\left.A \subseteq \Gamma_{1} \bigcap \Gamma_{2} \text { and }|A|=K(d)\right\},
\end{gathered}
$$

then we have the following lemma.

\section{Lemma 4.2.}

$$
\lim _{d \rightarrow+\infty} m(d)=1 .
$$

The proof of Lemma 4.2 is given in Subsection 4.3.

Now we show how to utilize Lemmas 4.1 and 4.2 to prove Equation (2.3).

Proof of Equation (2.3): For $x, y \in \mathbb{Z}_{+}^{d}$, we write $x \rightrightarrows y$ when there exists

$$
x_{1}, x_{2}, \ldots, x_{m}
$$

for some integer $m \geq 1$ such that $x=x_{0} \rightarrow x_{1} \rightarrow x_{2} \rightarrow \ldots \rightarrow x_{m} \rightarrow x_{m+1}=y$ and $\widetilde{U}\left(x_{j}, x_{j+1}\right)<\widetilde{Y}\left(x_{j}\right)$ for all $0 \leq j \leq m$. Then, according to the meaning of the exponential times $\widetilde{U}(\cdot, \cdot)$ and $\widetilde{Y}(\cdot)$,

$$
\bigcup_{t \geq 0} I_{t}^{A}=A \cup\{y: x \rightrightarrows y \text { for some } x \in A\} .
$$

Since each infected vertex becomes recovered in an exponential time with rate one, the infected vertices never die out when and only when there are infinitely many vertices which have ever been infected. Therefore,

$$
\left\{I_{t}^{A} \neq \emptyset, \forall t \geq 0\right\}=\{\mid\{y: x \rightrightarrows y \text { for some } x \in A\} \mid=+\infty\}
$$


for any finite $A$. According to the definition of $V_{\left\lfloor\sigma_{0} \log d\right\rfloor}, O \rightrightarrows x$ for any $x \in$ $V_{\left\lfloor\sigma_{0} \log d\right\rfloor}$. As a result,

$$
\left\{y: x \rightrightarrows y \text { for some } x \in V_{\left\lfloor\sigma_{0} \log d\right\rfloor}\right\} \subseteq\{y: O \rightrightarrows y\} \subseteq \bigcup_{t \geq 0} I_{t}^{O},
$$

since $O \rightrightarrows y$ when $O \rightrightarrows x$ and $x \rightrightarrows y$. By Equations (4.2) and (4.3),

$$
P_{\lambda, d}\left(\mid\left\{y: x \rightrightarrows y \text { for some } x \in V_{\left\lfloor\sigma_{0} \log d\right\rfloor}\right\} \mid=+\infty\right) \leq P_{\lambda, d}\left(I_{t}^{O} \neq \emptyset, \forall t \geq 0\right) .
$$

According to the conditional probability formula,

$$
\begin{aligned}
& P_{\lambda, d}\left(\mid\left\{y: x \rightrightarrows y \text { for some } x \in V_{\left\lfloor\sigma_{0} \log d\right\rfloor}\right\} \mid=+\infty\right) \\
& \geq P_{\lambda, d}\left(\mid\left\{y: x \rightrightarrows y \text { for some } x \in V_{\left\lfloor\sigma_{0} \log d\right\rfloor}\right\}|=+\infty|\left|V_{\left\lfloor\sigma_{0} \log d\right\rfloor}\right| \geq K(d)\right) \\
& \times P_{\lambda, d}\left(\left|V_{\left\lfloor\sigma_{0} \log d\right\rfloor}\right| \geq K(d)\right) .
\end{aligned}
$$

We define $\widetilde{h}(d)$ as

$$
\widetilde{h}(d)=\left\{A: A \subseteq \Gamma_{1} \bigcap \Gamma_{2} \text { and }|A| \geq K(d)\right\},
$$

then by Equation (4.2),

$$
\begin{aligned}
& P_{\lambda, d}\left(\mid\left\{y: x \rightrightarrows y \text { for some } x \in V_{\left\lfloor\sigma_{0} \log d\right\rfloor}\right\}|=+\infty|\left|V_{\left\lfloor\sigma_{0} \log d\right\rfloor}\right| \geq K(d)\right) \\
& =\sum_{A \in \widetilde{h}(d)} P_{\lambda, d}\left(\mid\left\{y: x \rightrightarrows y \text { for some } x \in V_{\left\lfloor\sigma_{0} \log d\right\rfloor}\right\} \mid=+\infty,\right. \\
& \left.V_{\left\lfloor\sigma_{0} \log d\right\rfloor}=A|| V_{\left\lfloor\sigma_{0} \log d\right\rfloor} \mid \geq K(d)\right) \\
& =\sum_{A \in \widetilde{h}(d)} P_{\lambda, d}\left(I_{t}^{A} \neq \emptyset \text { for all } t \geq 0 \mid V_{\left\lfloor\sigma_{0} \log d\right\rfloor}=A\right) \\
& \quad \times P_{\lambda, d}\left(V_{\left\lfloor\sigma_{0} \log d\right\rfloor}=A|| V_{\left\lfloor\sigma_{0} \log d\right\rfloor} \mid \geq K(d)\right) .
\end{aligned}
$$

For any $x \in V_{\left\lfloor\sigma_{0} \log d\right\rfloor}, \rho(x)>0$ since $x$ can be infected. Then, by Assumption (4.1), $\rho(x) \geq \epsilon$ for any $x \in V_{\left\lfloor\sigma_{0} \log d\right\rfloor}$. As a result,

$$
\begin{aligned}
& P_{\lambda, d}\left(I_{t}^{A} \neq \emptyset \text { for all } t \geq 0 \mid V_{\left\lfloor\sigma_{0} \log d\right\rfloor}=A\right) \\
& \geq P_{\lambda, d}\left(I_{t}^{A} \neq \emptyset \text { for all } t \geq 0 \mid V_{\left\lfloor\sigma_{0} \log d\right\rfloor}=A, \rho(x)=\epsilon \text { for all } x \in A\right) .
\end{aligned}
$$

Conditioned on $\{\rho(x)=\epsilon$ for all $x \in A\}$ for some $A \in \widetilde{h}(d)$, the event $\left\{I_{t}^{A} \neq\right.$ $\emptyset$ for all $t \geq 0\}$ is independent of $\left\{V_{\left\lfloor\sigma_{0} \log d\right\rfloor}=A\right\}$, since $\left\{I_{t}^{A}\right\}_{t \geq 0}$ only depends on

$$
\wp_{1}=\left\{\widetilde{U}(x, y): x \in A \text { or }\|x\|>\left\lfloor\sigma_{0} \log d\right\rfloor, x \rightarrow y\right\} \bigcup\left\{\widetilde{Y}(x): x \in \Gamma_{2}\right\}
$$

and $V_{\left\lfloor\sigma_{0} \log d\right\rfloor}$ only depends on

$$
\wp_{2}=\left\{\widetilde{U}(x, y):\|x\|<\left\lfloor\sigma_{0} \log d\right\rfloor, x \rightarrow y\right\} \bigcup\left\{\widetilde{Y}(x):\|x\|<\left\lfloor\sigma_{0} \log d\right\rfloor\right\}
$$

while $\wp_{1}$ and $\wp_{2}$ are independent when the values of $\{\rho(x)\}_{x \in A}$ are given. 
Therefore,

$$
\begin{aligned}
& P_{\lambda, d}\left(I_{t}^{A} \neq \emptyset \text { for all } t \geq 0 \mid V_{\left\lfloor\sigma_{0} \log d\right\rfloor}=A, \rho(x)=\epsilon \text { for all } x \in A\right) \\
& =P_{\lambda, d}\left(I_{t}^{A} \neq \emptyset \text { for all } t \geq 0 \mid \rho(x)=\epsilon \text { for all } x \in A\right) .
\end{aligned}
$$

It is obvious that

$$
\begin{aligned}
& P_{\lambda, d}\left(I_{t}^{A} \neq \emptyset \text { for all } t \geq 0 \mid \rho(x)=\epsilon \text { for all } x \in A\right) \\
& \geq P_{\lambda, d}\left(I_{t}^{B} \neq \emptyset \text { for all } t \geq 0 \mid \rho(x)=\epsilon \text { for all } x \in B\right)
\end{aligned}
$$

for $B \subseteq A \subseteq \Gamma_{1} \bigcap \Gamma_{2}$. As a result,

$$
P_{\lambda, d}\left(I_{t}^{A} \neq \emptyset \text { for all } t \geq 0 \mid \rho(x)=\epsilon \text { for all } x \in A\right) \geq m(d)
$$

for any $A \in \widetilde{h}(d)$, since each $A \in \widetilde{h}(d)$ has a subset with cardinality $K(d)$. By Equations (4.7), (4.8) and (4.9),

$$
P_{\lambda, d}\left(I_{t}^{A} \neq \emptyset \text { for all } t \geq 0 \mid V_{\left\lfloor\sigma_{0} \log d\right\rfloor}=A\right) \geq m(d)
$$

for each $A \in \widetilde{h}(d)$. Then, by Equation (4.6),

$$
\begin{aligned}
& P_{\lambda, d}\left(\mid\left\{y: x \rightrightarrows y \text { for some } x \in V_{\left\lfloor\sigma_{0} \log d\right\rfloor}\right\} \mid=+\infty\right) \\
& \geq m(d) \sum_{A \in \widetilde{h}(d)} P_{\lambda, d}\left(V_{\left\lfloor\sigma_{0} \log d\right\rfloor}=A|| V_{\left\lfloor\sigma_{0} \log d\right\rfloor} \mid \geq K(d)\right)=m(d) .
\end{aligned}
$$

By Equations (4.4), (4.5) and (4.11),

$$
P_{\lambda, d}\left(I_{t}^{O} \neq \emptyset, \forall t \geq 0\right) \geq m(d) P_{\lambda, d}\left(\left|V_{\left\lfloor\sigma_{0} \log d\right\rfloor}\right| \geq K(d)\right)
$$

and Equation (2.3) follows directly from Lemmas 4.1 and 4.2.

4.1. The coupling between $\left\{W_{n}\right\}_{n \geq 1}$ and $\left\{V_{n}\right\}_{n \geq 1}$. In this section, we give a coupling between the SIR model $\left\{V_{n}\right\}_{n \geq 0}$ on $\mathbb{Z}_{+}^{d}$ and the branching process $\left\{W_{n}\right\}_{n \geq 0}$ on $\mathbb{T}^{d}$.

We let $\{\rho(x)\}_{x \in \mathbb{Z}_{+}^{d}}$ be i.i.d copies of $\rho$ as defined in Section 1. We let $\{\widetilde{Y}(x)\}_{x \in \mathbb{Z}_{+}^{d}}$ and $\{\widetilde{U}(x, y)\}_{x \rightarrow y}$ be exponential times with respect to $\{\rho(x)\}_{x \in \mathbb{Z}_{+}^{d}}$ as defined at the beginning of this section. We let $\left\{V_{n}\right\}_{n \geq 0}$ be the SIR model with respect to $\tilde{Y}(\cdot)$ and $\widetilde{U}(\cdot, \cdot)$ as defined at the beginning of this section. Now we give the evolution of $\left\{W_{n}\right\}_{n \geq 0}$ by induction.

We let $W_{0}=\Upsilon, \rho(\Upsilon)=\rho(O)$ and $Y(\Upsilon)=\tilde{Y}(O)$, where $O$ is the origin of $\mathbb{Z}_{+}^{d}$. For the $d$ sons denoted by $n_{1}, n_{2}, \ldots, n_{d}$ of $\Upsilon$, we let $\rho\left(n_{i}\right)=\rho\left(e_{i}\right), Y\left(n_{i}\right)=\widetilde{Y}\left(e_{i}\right)$ and $U\left(\Upsilon, n_{i}\right)=\widetilde{U}\left(O, e_{i}\right)$ for each $1 \leq i \leq d$, where $e_{i}$ is the elementary unit vector of $\mathbb{Z}_{+}^{d}$ as defined in Section 1 . Then $W_{1}$ is defined according to the values of $\left\{U\left(\Upsilon, n_{i}\right)\right\}_{1 \leq i \leq d}$ and $Y(\Upsilon)$ as in Section 3.

For $n \geq 1$, if $\left|V_{n}\right|=\left|W_{n}\right|$ and there is a bijection $g_{n}: V_{n} \rightarrow W_{n}$ such that $\rho\left(g_{n}(x)\right)=\rho(x)$ and $Y\left(g_{n}(x)\right)=\tilde{Y}(x)$ for each $x \in V_{n}$, then we say that our coupling is successful at step $n$. It is obvious that our coupling is successful at step $n=1$ since $g_{1}$ can be defined as $g_{1}\left(e_{i}\right)=n_{i}$ for any $e_{i} \in V_{1}$. 
If $\left\{W_{m}\right\}_{m \leq n}$ is well defined and the coupling is successful at step $m$ for all $1 \leq m \leq n$, then $W_{n+1}$ is defined as follows. For any $x \in V_{n}$, we define

$$
\begin{gathered}
q(x)=\left\{y: x \rightarrow y \text { and } z \rightarrow y \text { for some } z \in V_{n} \backslash\{x\}\right\}, \\
\psi(x)=\{y: x \rightarrow y\} \backslash q(x)
\end{gathered}
$$

and $h(x)=d-|q(x)|$, then $|\psi(x)|=h(x)$. For each $x \in V_{n}$, we arbitrarily choose $h(x)$ sons of $g_{n}(x) \in W_{n}$, which are denoted by $w_{1}, w_{2}, \ldots, w_{h(x)}$. Giving the $h(x)$ elements in $\psi(x)$ an arbitrary order $y_{1}, y_{2}, \ldots, y_{h(x)}$, then we let $\rho\left(w_{i}\right)=\rho\left(y_{i}\right)$, $Y\left(w_{i}\right)=\tilde{Y}\left(y_{i}\right)$ and $U\left(g_{n}(x), w_{i}\right)=\widetilde{U}\left(x, y_{i}\right)$ for each $1 \leq i \leq h(x)$. For any son $u$ of $g_{n}(x)$ which is not in $\left\{w_{1}, w_{2}, \ldots, w_{h(x)}\right\}$, let $Y(u)$ be an exponential time with rate one and $\rho(u)$ be an independent copy of $\rho$ such that $Y(u)$ and $\rho(u)$ are independent of the aforesaid exponential times and vertex weights while let $U\left(g_{n}(x), u\right)$ be an exponential time with rate $\frac{\lambda}{d} \rho\left(g_{n}(x)\right) \rho(u)$. Then, $W_{n+1}$ is defined according to the values of $\left\{Y\left(g_{n}(x)\right)\right\}_{x \in V_{n}}$ and $\left\{U\left(g_{n}(x), w\right)\right\}_{x \in V_{n}, g_{n}(x) \Rightarrow w}$ as in Section 3.

If $n$ is the first step that the coupling is not successful, then we let $\left\{W_{m}\right\}_{m \geq n+1}$ evolve independently of $\left\{V_{m}\right\}_{m \geq n+1}$.

From now on we assume that $\left\{W_{n}\right\}_{n \geq 0}$ and $\left\{V_{n}\right\}_{n \geq 0}$ are defined under the same probability space. The annealed measure is still denoted by $P_{\lambda, d}$.

The remainder of this subsection is devoted to the proof of the following lemma.

Lemma 4.3. We denote by $B(d)$ the event that the coupling of $\left\{V_{n}\right\}_{n \geq 0}$ on $\mathbb{Z}_{+}^{d}$ and $\left\{W_{n}\right\}_{n \geq 0}$ on $\mathbb{T}^{d}$ is successful at step $m$ for all

$$
1 \leq m \leq\left\lfloor\sigma_{0} \log d\right\rfloor
$$

then

$$
\lim _{d \rightarrow+\infty} P_{\lambda, d}(B(d))=1 .
$$

Proof of Lemma 4.3: First we claim that

$$
P_{\lambda, d}\left(\left|V_{m}\right|>d^{0.2}\right) \leq \frac{d^{-0.1} \lambda M^{2}}{\lambda M^{2}-1}
$$

for each $0 \leq m \leq\left\lfloor\sigma_{0} \log d\right\rfloor$. Equation (4.12) follows from the following analysis. For a given oriented path $\vec{l}: O=x_{0} \rightarrow x_{1} \rightarrow \ldots \rightarrow x_{m}$ on $\mathbb{Z}_{+}^{d}$,

$$
P_{\lambda, d}\left(\widetilde{U}\left(x_{j}, x_{j+1}\right)<\widetilde{Y}\left(x_{j}\right) \text { for all } 0 \leq j \leq m-1\right) \leq\left(\frac{\lambda}{d} M^{2}\right)^{m},
$$

since $\widetilde{Y}(\cdot)$ is an exponential time with rate one while $\widetilde{U}(\cdot, \cdot)$ is an exponential time with rate at most $\frac{\lambda}{d} M^{2}$. The number of oriented paths starting at $O$ with length $m$ on $\mathbb{Z}_{d}^{+}$is $d^{m}$. As a result,

$$
E_{\lambda, d}\left|V_{m}\right| \leq\left(\frac{\lambda}{d} M^{2}\right)^{m} d^{m}=\left(\lambda M^{2}\right)^{m},
$$

since $x \in V_{m}$ when and only when there exists an oriented path $\vec{l}: O=x_{0} \rightarrow x_{1} \rightarrow$ $\ldots \rightarrow x_{m}=x$ that $\widetilde{U}\left(x_{j}, x_{j+1}\right)<\widetilde{Y}\left(x_{j}\right)$ for all $0 \leq j \leq m-1$.

Then, according to the Chebyshev's inequality and the fact that $\sigma_{0} \log \left(\lambda M^{2}\right)<$ $\frac{1}{10}$

$$
P_{\lambda, d}\left(\sum_{m=0}^{\left\lfloor\sigma_{0} \log d\right\rfloor}\left|V_{m}\right|>d^{0.2}\right) \leq d^{-0.2} \sum_{m=0}^{\left\lfloor\sigma_{0} \log d\right\rfloor}\left(\lambda M^{2}\right)^{m} \leq \frac{d^{-0.1} \lambda M^{2}}{\lambda M^{2}-1},
$$

and Equation (4.12) follows from which directly. 
For $1 \leq m \leq\left\lfloor\sigma_{0} \log d\right\rfloor$, we use $B(d, m)$ to denote the event that the coupling of $\left\{V_{n}\right\}_{n \geq 0}$ on $\mathbb{Z}_{+}^{d}$ and $\left\{W_{n}\right\}_{n \geq 0}$ on $\mathbb{T}^{d}$ is successful at step $l$ for all $1 \leq l \leq m$. Then $B(d)=B\left(d,\left\lfloor\sigma_{0} \log d\right\rfloor\right)$ and $P_{\lambda, d}(B(d, 1))=1$.

For $d \geq 2$ and $1 \leq m \leq\left\lfloor\sigma_{0} \log d\right\rfloor-1$, we denote by $J(d, m)$ the event that $\widetilde{U}(x, y)>\widetilde{Y}(x)$ for any $x \in V_{m}$ and any $y \in q(x)$. We use $J(d)$ to denote $\bigcap_{m=1}^{\left\lfloor\sigma_{0} \log d\right\rfloor-1} J(d, m)$, i.e., $J(d)$ is the event that $\widetilde{U}(x, y)>\widetilde{Y}(x)$ for any $x \in \bigcup_{m=0}^{\left\lfloor\sigma_{0} \log d\right\rfloor-1} V_{m}$ and any $y \in q(x)$.

It is easy to check that there exists a vertex $y$ satisfying $x \rightarrow y, z \rightarrow y$ for given $x, z \in V_{m}$ when and only when $x-z=e_{i}-e_{j}$ for some $1 \leq i, j \leq d$ and such $y$ is unique that $y=x+e_{j}=z+e_{i}$. Hence,

$$
|q(x)| \leq\left|V_{m}\right|-1<\left|V_{m}\right|
$$

for any $x \in V_{m}$. For $k<\left\lfloor\sigma_{0} \log d\right\rfloor$, conditioned on $B(d, k)$, the coupling will be successful at step $k+1$ if $J(d, k)$ occurs and $U\left(g_{k}(x), y\right)>Y\left(g_{k}(x)\right)$ for any $x \in V_{k}$ and any $y$ that $g_{k}(x) \Rightarrow y$ while $y \neq w_{1}, w_{2}, \ldots, w_{h(x)}$. Then, by Equation (4.13) and the fact that $\tilde{Y}(\cdot), Y(\cdot)$ are exponential times with rates 1 while $\widetilde{U}(\cdot, \cdot), U(\cdot, \cdot)$ are exponential times with rates at most $\frac{\lambda}{d} M^{2}$,

$$
P_{\lambda, d}\left(B(d, k+1)|B(d, k),| V_{k} \mid \leq d^{0.2}\right) \geq 1-2 d^{0.2} \times d^{0.2} \frac{\lambda M^{2}}{d}=1-2 \lambda M^{2} d^{-0.6}
$$

By Equations (4.12) and (4.14),

$$
\begin{aligned}
P_{\lambda, d}(B(d, k+1)) \geq & P_{\lambda, d}\left(B(d, k+1), B(d, k),\left|V_{k}\right| \leq d^{0.2}\right) \\
\geq & P_{\lambda, d}\left(B(d, k+1)|B(d, k),| V_{k} \mid \leq d^{0.2}\right) \\
& \times\left[P_{\lambda, d}(B(d, k))-P\left(\left|V_{k}\right|>d^{0.2}\right)\right] \\
\geq & \left(1-2 \lambda M^{2} d^{-0.6}\right)\left[P_{\lambda, d}(B(d, k))-\frac{d^{-0.1} \lambda M^{2}}{\lambda M^{2}-1}\right] .
\end{aligned}
$$

By Equation (4.15),

$$
\begin{aligned}
& P_{\lambda, d}(B(d, k+1))+\frac{\left(1-2 \lambda M^{2} d^{-0.6}\right) d^{0.5}}{2\left(\lambda M^{2}-1\right)} \\
& \geq\left(1-2 \lambda M^{2} d^{-0.6}\right)\left[P_{\lambda, d}(B(d, k))+\frac{\left(1-2 \lambda M^{2} d^{-0.6}\right) d^{0.5}}{2\left(\lambda M^{2}-1\right)}\right]
\end{aligned}
$$

and hence

$$
\begin{aligned}
P_{\lambda, d}(B(d, k)) \geq & \left(1-2 \lambda M^{2} d^{-0.6}\right)^{k-1}\left[1+\frac{\left(1-2 \lambda M^{2} d^{-0.6}\right) d^{0.5}}{2\left(\lambda M^{2}-1\right)}\right] \\
& -\frac{\left(1-2 \lambda M^{2} d^{-0.6}\right) d^{0.5}}{2\left(\lambda M^{2}-1\right)}
\end{aligned}
$$

for $1 \leq k \leq\left\lfloor\sigma_{0} \log d\right\rfloor$, since $P_{\lambda, d}(B(d, 1))=1$. 
Lemma 4.3 follows from Equation (4.16) directly since $B(d)=B\left(d,\left\lfloor\sigma_{0} \log d\right\rfloor\right)$ and

$$
\begin{aligned}
\lim _{d \rightarrow+\infty} & \left\{\left(1-2 \lambda M^{2} d^{-0.6}\right)^{\left\lfloor\sigma_{0} \log d\right\rfloor-1}\left[1+\frac{\left(1-2 \lambda M^{2} d^{-0.6}\right) d^{0.5}}{2\left(\lambda M^{2}-1\right)}\right]\right. \\
& \left.-\frac{\left(1-2 \lambda M^{2} d^{-0.6}\right) d^{0.5}}{2\left(\lambda M^{2}-1\right)}\right\}=1 .
\end{aligned}
$$

4.2. Proof of Lemma 4.1. In this subsection we give the proof of Lemma 4.1. As a preparation, we introduce some notations and definitions. For sufficiently large $d$, let $N(d)=\log (\log d)$ as we have introduced. For each $x \in \mathbb{T}^{d}$, we give the $d$ sons of $x$ an order $x(1), x(2), \ldots, x(d)$, i.e., $x(i)$ is the $i$ th son of $x$. Then, we define

(1) $\widehat{W}_{0}=\Upsilon$.

(2) For each $n \geq 0$,

$$
\begin{aligned}
\widehat{W}_{n+1}= & \left\{y \text { : there exists } x \in \widehat{W}_{n} \text { that } y=x(i)\right. \\
& \text { for some } \left.i \leq d-\left\lfloor\frac{d}{N(d)}\right\rfloor \text { and } U(x, y)<Y(x)\right\} .
\end{aligned}
$$

It is obvious that $\widehat{W}_{n} \subseteq W_{n}$ for each $n \geq 0$. We define

$$
\begin{aligned}
& \widehat{D}=\left\{y: \text { there exists } x \in \bigcup_{m=0}^{\left\lfloor\sigma_{0} \log d\right\rfloor-1} \widehat{W}_{m}\right. \\
& \quad \text { such that } y=x(i) \text { for some } i \geq j(d) \text { and } U(x, y)<Y(x)\},
\end{aligned}
$$

where $j(d)=d-\left\lfloor\frac{d}{N(d)}\right\rfloor+1$ as we have introduced. The proof of Lemma 4.1 relies heavily on Lemma 4.3 and the following lemma.

\section{Lemma 4.4.}

$$
\lim _{d \rightarrow+\infty} P_{\lambda, d}\left(|\widehat{D}| \geq\left\lfloor\frac{\sqrt{\log d}}{N(d)}\right\rfloor \mid \widehat{W}_{\left\lfloor\sigma_{0} \log d\right\rfloor-1} \neq \emptyset\right)=1 .
$$

Note that we use $P_{\lambda, d}$ instead of $\widehat{P}_{\lambda, d}$ in Lemma 4.4 since we have already coupled $\left\{W_{n}\right\}_{n \geq 1}$ with $\left\{V_{n}\right\}_{n \geq 1}$. The proof of Lemma 4.4 is given in the next subsection. Now we give the proof of Lemma 4.1.

Proof of Lemma 4.1: For each $u \in \mathbb{T}^{d}$, we denote by $\mathbb{T}_{u}$ the subtree of $\mathbb{T}^{d}$ rooted at $u$ and consisted of $u$ and its descendants. We denote by $\chi(u)$ the indicator function of the event that the infected vertices in the SIR model confined on $\mathbb{T}_{u}$ with $u$ being initially infected while others being initially susceptible never die out. For each $u \in \widehat{D}$, since $u$ has been infected by $\Upsilon$ through a path from $\Upsilon$ to $u, \rho(u)>0$ and hence $\rho(u) \geq \epsilon$ according to Assumption 4.1. For any $u, v \in \widehat{D}$, it is easy to check that

$$
\mathbb{T}_{u} \cap \mathbb{T}_{v}=\emptyset \quad \text { and } \quad \mathbb{T}_{u} \cap\left(\bigcup_{n \leq\left\lfloor\sigma_{0} \log d\right\rfloor-1} \widehat{W}_{n}\right)=\emptyset .
$$

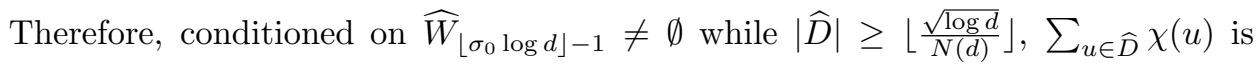
stochastic dominated from below by a random variable $\widetilde{D}(d)$ following from the 
binomial distribution $B\left(\left\lfloor\frac{\sqrt{\log d}}{N(d)}\right\rfloor, 1-F_{d}(\epsilon)\right)$, where

$$
F_{d}(\epsilon)=P_{\lambda, d}\left(W_{n}=\emptyset \text { for some } n \geq 0 \mid \rho(\Upsilon)=\epsilon\right)
$$

defined as in Section 3. According to the proof of Lemma 3.1,

$$
\lim _{d \rightarrow+\infty} 1-F_{d}(\epsilon)=\frac{\lambda \epsilon \theta}{(1+\lambda \epsilon \theta)}
$$

and hence

$$
\lim _{d \rightarrow \infty} \frac{\widetilde{D}(d) N(d)}{\sqrt{\log d}}=\frac{\lambda \epsilon \theta}{(1+\lambda \epsilon \theta)}
$$

in probability by the law of large numbers. As a result,

$$
\begin{aligned}
& \liminf _{d \rightarrow+\infty} P_{\lambda, d}\left(\sum_{u \in \widehat{D}} \chi(u) \geq K(d)\left|\widehat{W}_{\left\lfloor\sigma_{0} \log d\right\rfloor-1} \neq \emptyset,\right| \widehat{D} \mid \geq\left\lfloor\frac{\sqrt{\log d}}{N(d)}\right\rfloor\right) \\
& \left.=\liminf _{d \rightarrow+\infty} P_{\lambda, d}\left(\sum_{u \in \widehat{D}} \chi(u) \geq\left\lfloor\frac{\lambda \epsilon \theta}{2(1+\lambda \epsilon \theta)} \frac{\sqrt{\log d}}{N(d)}\right\rfloor \mid \widehat{W}_{\left\lfloor\sigma_{0}\right.} \log d\right\rfloor-1 \neq \emptyset,|\widehat{D}| \geq\left\lfloor\frac{\sqrt{\log d}}{N(d)}\right\rfloor\right) \\
& \geq \liminf _{d \rightarrow+\infty} P\left(\widetilde{D}(d) \geq\left\lfloor\frac{\lambda \epsilon \theta}{2(1+\lambda \epsilon \theta)} \frac{\sqrt{\log d}}{N(d)}\right\rfloor\right)=1 .
\end{aligned}
$$

For any $u \in \widehat{D}$, if $\chi(u)=1$, since $u$ has been infected while the distance between $\Upsilon$ and $u$ is at most $\left\lfloor\sigma_{0} \log d\right\rfloor$, then there exists at least one vertex $z \in \mathbb{T}_{u}$ such that the distance between $\Upsilon$ and $z$ is $\left\lfloor\sigma_{0} \log d\right\rfloor$ while $z$ has been infected, i.e.,

$$
z \in W_{\left\lfloor\sigma_{0} \log d\right\rfloor} \text {. }
$$

Since $\mathbb{T}_{u} \cap \mathbb{T}_{v}=\emptyset$ for any $u, v \in \widehat{D}$,

$$
\left\{\sum_{u \in \widehat{D}} \chi(u) \geq K(d)\right\} \subseteq\left\{\left|W_{\left\lfloor\sigma_{0} \log d\right\rfloor}\right| \geq K(d)\right\} .
$$

Then, by Equation (4.17),

$$
\liminf _{d \rightarrow+\infty} P_{\lambda, d}\left(\left|W_{\left\lfloor\sigma_{0} \log d\right\rfloor}\right| \geq K(d)\left|\widehat{W}_{\left\lfloor\sigma_{0} \log d\right\rfloor-1} \neq \emptyset,\right| \widehat{D} \mid \geq\left\lfloor\frac{\sqrt{\log d}}{N(d)}\right\rfloor\right)=1 .
$$

Then, by Lemma 4.4 and Equation (4.18),

$$
\begin{aligned}
& \liminf _{d \rightarrow+\infty} P_{\lambda, d}\left(\left|W_{\left\lfloor\sigma_{0} \log d\right\rfloor}\right| \geq K(d) \mid \widehat{W}_{\left\lfloor\sigma_{0} \log d\right\rfloor-1} \neq \emptyset\right) \\
& \geq \liminf _{d \rightarrow+\infty} P_{\lambda, d}\left(\left|W_{\left\lfloor\sigma_{0} \log d\right\rfloor}\right| \geq K(d),|\widehat{D}| \geq\left\lfloor\frac{\sqrt{\log d}}{N(d)}\right\rfloor \mid \widehat{W}_{\left\lfloor\sigma_{0} \log d\right\rfloor-1} \neq \emptyset\right) \\
& =\liminf _{d \rightarrow+\infty} P_{\lambda, d}\left(\left|W_{\left\lfloor\sigma_{0} \log d\right\rfloor}\right| \geq K(d)\left|\widehat{W}_{\left\lfloor\sigma_{0} \log d\right\rfloor-1} \neq \emptyset,\right| \widehat{D} \mid \geq\left\lfloor\frac{\sqrt{\log d}}{N(d)}\right\rfloor\right) \\
& \quad \times P_{\lambda, d}\left(|\widehat{D}| \geq\left\lfloor\frac{\sqrt{\log d}}{N(d)}\right\rfloor \mid \widehat{W}_{\left\lfloor\sigma_{0} \log d\right\rfloor-1} \neq \emptyset\right) \\
& =1 .
\end{aligned}
$$

According to the definition of $\left\{\widehat{W}_{n}\right\}_{n \geq 0},\left\{\widehat{W}_{n}\right\}_{n \geq 0}$ on $\mathbb{T}^{d}$ with infection rate $\lambda$ can be identified with $\left\{W_{n}\right\}_{n \geq 0}$ on $\mathbb{T}^{d-\left\lfloor\frac{d}{N(d)}\right\rfloor}$ with infection rate

$$
\widetilde{\lambda}(d)=\lambda \frac{d-\left\lfloor\frac{d}{N(d)}\right\rfloor}{d} .
$$


For given $\lambda_{1} \in\left(\frac{1}{E\left(\rho^{2}\right)}, \lambda\right)$,

$$
\widetilde{\lambda}(d) \geq \lambda_{1}
$$

for sufficiently large $d$. Hence, by Lemma 3.1,

$$
\begin{aligned}
& \liminf _{d \rightarrow+\infty} P_{\lambda, d}\left(\widehat{W}_{\left\lfloor\sigma_{0} \log d-1\right\rfloor} \neq \emptyset\right) \geq \liminf _{d \rightarrow+\infty} P_{\lambda, d}\left(\widehat{W}_{n} \neq \emptyset \text { for all } n \geq 0\right) \\
& =\liminf _{d \rightarrow+\infty} P_{\widetilde{\lambda}(d), d-\left\lfloor\frac{d}{N(d)}\right\rfloor}\left(W_{n} \neq \emptyset \text { for all } n \geq 0\right) \\
& \geq \liminf _{d \rightarrow+\infty} P_{\lambda_{1}, d-\left\lfloor\frac{d}{N(d)}\right\rfloor}\left(W_{n} \neq \emptyset \text { for all } n \geq 0\right)=E\left(\frac{\lambda_{1} \rho \theta_{1}}{1+\lambda_{1} \rho \theta_{1}}\right),
\end{aligned}
$$

where $\theta_{1}=\theta_{1}\left(\lambda_{1}\right)$ is the unique solution of

$$
E\left(\frac{\lambda_{1} \rho^{2}}{1+\lambda_{1} \rho \theta_{1}}\right)=1
$$

It is easy to check that $\lim _{\lambda_{1} \uparrow \lambda} \theta_{1}=\theta$ while

$$
\lim _{\lambda_{1} \uparrow \lambda} E\left(\frac{\lambda_{1} \rho \theta_{1}}{1+\lambda_{1} \rho \theta_{1}}\right)=E\left(\frac{\lambda \rho \theta}{1+\lambda \rho \theta}\right) .
$$

Hence, let $\lambda_{1} \uparrow \lambda$, we have

$$
\liminf _{d \rightarrow+\infty} P_{\lambda, d}\left(\widehat{W}_{\left\lfloor\sigma_{0} \log d\right\rfloor-1} \neq \emptyset\right) \geq E\left(\frac{\lambda \rho \theta}{1+\lambda \rho \theta}\right) .
$$

By Equations (4.19), (4.20) and the conditional probability formula,

$$
\liminf _{d \rightarrow+\infty} P_{\lambda, d}\left(\left|W_{\left\lfloor\sigma_{0} \log d\right\rfloor}\right| \geq K(d)\right) \geq E\left(\frac{\lambda \rho \theta}{1+\lambda \rho \theta}\right) .
$$

By Equation (4.21), Lemma 4.3 and the fact that $\left|V_{\left\lfloor\sigma_{0} \log d\right\rfloor}\right|=\left|W_{\left\lfloor\sigma_{0} \log d\right\rfloor}\right|$ on the event $B(d)$,

$$
\begin{aligned}
& \liminf _{d \rightarrow+\infty} P_{\lambda, d}\left(\left|V_{\left\lfloor\sigma_{0} \log d\right\rfloor}\right| \geq K(d)\right) \geq \liminf _{d \rightarrow+\infty} P_{\lambda, d}\left(\left|V_{\left\lfloor\sigma_{0} \log d\right\rfloor}\right| \geq K(d), B(d)\right) \\
& =\liminf _{d \rightarrow+\infty} P_{\lambda, d}\left(\left|W_{\left\lfloor\sigma_{0} \log d\right\rfloor}\right| \geq K(d), B(d)\right) \\
& \geq \liminf _{d \rightarrow+\infty} P_{\lambda, d}\left(\left|W_{\left\lfloor\sigma_{0} \log d\right\rfloor}\right| \geq K(d)\right)-\lim _{d \rightarrow+\infty} P_{\lambda, d}\left(B(d)^{c}\right) \\
& \geq E\left(\frac{\lambda \rho \theta}{1+\lambda \rho \theta}\right)-0=E\left(\frac{\lambda \rho \theta}{1+\lambda \rho \theta}\right)
\end{aligned}
$$

and the proof is complete.

4.3. Proof of Lemma 4.4. In this section we give the proof of Lemma 4.4. First we introduce some notations and definitions.

We let $\widehat{Y}_{0}, \ldots, \widehat{Y}_{\left\lfloor\sigma_{0} \log d\right\rfloor-1}$ be exponential times with rate one while

$$
\Lambda_{0}, \ldots, \Lambda_{\left\lfloor\sigma_{0} \log d\right\rfloor-1}
$$

be exponential times with rate $\lambda M^{2}$. For $0 \leq i \leq\left\lfloor\sigma_{0} \log d\right\rfloor-1$ and $1 \leq j \leq\left\lfloor\frac{d}{N(d)}\right\rfloor$, let $\rho_{i j}$ be an independent copy of $\rho$ while $\widehat{U}_{i j}$ is an exponential time with rate $\frac{\lambda}{d} \epsilon \rho_{i j}$, where $\epsilon$ is defined as in Equation (4.1). According to the basic technique of measure theory, we can assume that $\left\{\rho_{i j}: 0 \leq i \leq\left\lfloor\sigma_{0} \log d\right\rfloor-1,1 \leq j \leq\left\lfloor\frac{d}{N(d)}\right\rfloor\right\}$ and $\{\rho(x)\}_{x \in \mathbb{T}^{d}}$ are defined under the same space and independent under the annealed 
measure $P_{\lambda, d}$ while we assume that $U(\cdot, \cdot), \widehat{U} . ., Y(\cdot), \widehat{Y} ., \Lambda$. are defined under the same space and independent under the quenched measure $P_{\lambda, \omega}$.

Lemma 4.5. Let $\xi_{i}=\sum_{j=1}^{\left\lfloor\frac{d}{N(d)}\right\rfloor} 1_{\left\{\widehat{U}_{i j}<\widehat{Y}_{i}\right\}}$ for $0 \leq i \leq\left\lfloor\sigma_{0} \log d\right\rfloor-1$, then

$$
\begin{aligned}
& P_{\lambda, d}\left(|\widehat{D}| \geq\left\lfloor\frac{\sqrt{\log d}}{N(d)}\right\rfloor \mid \widehat{W}_{\left\lfloor\sigma_{0} \log d\right\rfloor-1} \neq \emptyset\right) \\
& \geq P_{\lambda, d}\left(\sum_{i=0}^{\left\lfloor\sigma_{0} \log d\right\rfloor-1} \xi_{i} \geq\left\lfloor\frac{\sqrt{\log d}}{N(d)}\right\rfloor \mid \widehat{Y}_{i}<\Lambda_{i} \text { for all } 0 \leq i \leq\left\lfloor\sigma_{0} \log d\right\rfloor-1\right),
\end{aligned}
$$

and

$$
\lim _{d \rightarrow+\infty} E_{\lambda, d}\left(e^{-s \frac{N(d)}{\log d} \sum_{j=0}^{\left\lfloor\sigma_{0} \log d\right\rfloor-1} \xi_{j}} \mid \widehat{Y}_{i}<\Lambda_{i} \text { for all } 0 \leq i \leq\left\lfloor\sigma_{0} \log d\right\rfloor-1\right)
$$

exists for any $s>0$. Furthermore, we use $\Theta(s)$ to denote

$$
\lim _{d \rightarrow+\infty} E_{\lambda, d}\left(e^{-s \frac{N(d)}{\log d} \sum_{j=0}^{\left\lfloor\sigma_{0} \log d\right\rfloor-1} \xi_{j}} \mid \widehat{Y}_{i}<\Lambda_{i} \text { for all } 0 \leq i \leq\left\lfloor\sigma_{0} \log d\right\rfloor-1\right)
$$

then $\lim _{s \rightarrow+\infty} \Theta(s)=0$.

We give the proof of Lemma 4.5 at the end of this subsection. Now we show how to utilize Lemma 4.5 to prove Lemma 4.4 .

Proof of Lemma 4.4: By Chebyshev's inequality, for any $s>0$,

$$
\begin{aligned}
& P_{\lambda, d}\left(\sum_{i=0}^{\left\lfloor\sigma_{0} \log d\right\rfloor-1} \xi_{i} \leq\left\lfloor\frac{\sqrt{\log d}}{N(d)}\right\rfloor \mid \widehat{Y}_{i}<\Lambda_{i} \text { for all } 0 \leq i \leq\left\lfloor\sigma_{0} \log d\right\rfloor-1\right) \\
& \leq e^{s\left\lfloor\frac{\sqrt{\log d}}{N(d)}\right\rfloor \frac{N(d)}{\log d}} E_{\lambda, d}\left(e^{-s \frac{N(d)}{\log d}} \sum_{j=0}^{\left\lfloor\sigma_{0} \log d\right\rfloor-1} \xi_{j} \mid \widehat{Y}_{i}<\Lambda_{i} \text { for all } 0 \leq i \leq\left\lfloor\sigma_{0} \log d\right\rfloor-1\right) .
\end{aligned}
$$

Then, according to Equation (4.23),

$\limsup _{d \rightarrow+\infty} P_{\lambda, d}\left(\sum_{i=0}^{\left\lfloor\sigma_{0} \log d\right\rfloor-1} \xi_{i} \leq\left\lfloor\frac{\sqrt{\log d}}{N(d)}\right\rfloor \mid \widehat{Y}_{i}<\Lambda_{i}\right.$ for all $\left.0 \leq i \leq\left\lfloor\sigma_{0} \log d\right\rfloor-1\right) \leq \Theta(s)$

for any $s>0$, since $\lim _{d \rightarrow+\infty}\left(\left\lfloor\frac{\sqrt{\log d}}{N(d)}\right\rfloor \frac{N(d)}{\log d}\right)=0$.

Let $s \rightarrow+\infty$, since $\lim _{s \rightarrow+\infty} \Theta(s)=0$, we have

$$
\lim _{d \rightarrow+\infty} P_{\lambda, d}\left(\sum_{i=0}^{\left\lfloor\sigma_{0} \log d\right\rfloor-1} \xi_{i} \leq\left\lfloor\frac{\sqrt{\log d}}{N(d)}\right\rfloor \mid \widehat{Y}_{i}<\Lambda_{i} \text { for all } 0 \leq i \leq\left\lfloor\sigma_{0} \log d\right\rfloor-1\right)=0 .
$$

Lemma 4.4 follows from Equations (4.22) and (4.24) directly.

To give the proof of Lemma 4.5, we define a total order $\prec$ on $\widehat{W}_{n}$ for each $n \geq 1$. For any $u, v \in \widehat{W}_{n}, u \neq v$, there exists a unique common ancestor $x$ of $u, v$ such that $u \in \mathbb{T}_{x(i)}$ while $v \in \mathbb{T}_{x(j)}$ for some $1 \leq i \neq j \leq d-\left\lfloor\frac{d}{N(d)}\right\rfloor$. We write $u \prec v$ when and only when $i<j$. Now we give the proof of Lemma 4.5. 
Proof of Lemma 4.5: Equation (4.22) follows from the following analysis. Conditioned on $\widehat{W}_{\left\lfloor\sigma_{0} \log d\right\rfloor-1} \neq \emptyset$, there are $\Upsilon=X_{0} \Rightarrow X_{1} \Rightarrow \ldots \Rightarrow X_{\left\lfloor\sigma_{0} \log d\right\rfloor-1}$ such that $X_{i} \in \widehat{W}_{i}$ for $0 \leq i \leq\left\lfloor\sigma_{0} \log d\right\rfloor-1$. We choose $X_{1}, \ldots, X_{\left\lfloor\sigma_{0} \log d\right\rfloor-1}$ as follows. We let $X_{\left\lfloor\sigma_{0} \log d\right\rfloor-1}$ be the smallest one of $\widehat{W}_{\left\lfloor\sigma_{0} \log d\right\rfloor-1}$ under the partial $\prec$. Then, $\Upsilon=X_{0} \Rightarrow X_{1} \Rightarrow \ldots \Rightarrow X_{\left\lfloor\sigma_{0} \log d\right\rfloor-1}$ is the unique path on $\mathbb{T}^{d}$ from $O$ to $X_{\left\lfloor\sigma_{0} \log d\right\rfloor-1}$ with length $\left\lfloor\sigma_{0} \log d\right\rfloor-1$.

For $0 \leq i \leq\left\lfloor\sigma_{0} \log d\right\rfloor-1$, let

$$
\zeta_{i}=\sum_{j=1}^{\left\lfloor\frac{d}{N(d)}\right\rfloor}\left\{U\left(X_{i}, X_{i}\left(j+d-\left\lfloor\frac{d}{N(d)}\right\rfloor\right)\right)<Y\left(X_{i}\right)\right\} \text { and } \eta_{i}=\sum_{j=1}^{\left\lfloor\frac{d}{N(d)}\right\rfloor} 1_{\left\{\widehat{U}_{i j}<Y\left(X_{i}\right)\right\}},
$$

then

$$
|\widehat{D}| \geq \sum_{i=0}^{\left\lfloor\sigma_{0} \log d\right\rfloor-1} \zeta_{i}
$$

according to our definition of $\widehat{D}$. Note that $X_{i}(j)$ is the $j$ th son of $X_{i}$ according to the notations which we have introduced.

Since $X_{i} \in \widehat{W}_{i}, \rho\left(X_{i}\right)>0$ and hence $\rho\left(X_{i}\right) \geq \epsilon$ by assumption (4.1). As a result, $U\left(X_{i}, X_{i}\left(j+d-\left\lfloor\frac{d}{N(d)}\right\rfloor\right)\right)$ is an exponential time with rate at least $\frac{\lambda}{d} \epsilon \rho\left(X_{i}\left(j+d-\left\lfloor\frac{d}{N(d)}\right\rfloor\right)\right)$, where $\rho\left(X_{i}\left(j+d-\left\lfloor\frac{d}{N(d)}\right\rfloor\right)\right)$ is an independent copy of $\rho$. Therefore, $U\left(X_{i}, X_{i}\left(j+d-\left\lfloor\frac{d}{N(d)}\right\rfloor\right)\right)$ is stochastic dominated from above by $\widehat{U}_{i j}$ and $\eta_{i}$ is dominated from above by $\zeta_{i}$. Hence,

$$
\begin{aligned}
& P_{\lambda, d}\left(\sum_{i=0}^{\left\lfloor\sigma_{0} \log d\right\rfloor-1} \zeta_{i} \geq\left\lfloor\frac{\sqrt{\log d}}{N(d)}\right\rfloor \mid \widehat{W}_{\left\lfloor\sigma_{0} \log d\right\rfloor-1} \neq \emptyset\right) \\
& \geq P_{\lambda, d}\left(\sum_{i=0}^{\left\lfloor\sigma_{0} \log d\right\rfloor-1} \eta_{i} \geq\left\lfloor\frac{\sqrt{\log d}}{N(d)}\right\rfloor \mid \widehat{W}_{\left\lfloor\sigma_{0} \log d\right\rfloor-1} \neq \emptyset\right) .
\end{aligned}
$$

For any path $\vec{l}: O=l_{0} \Rightarrow l_{1} \Rightarrow \ldots \Rightarrow l_{\left\lfloor\sigma_{0} \log d\right\rfloor}$, we use $\gamma(\vec{l})$ to denote

$$
P_{\lambda, d}\left(X_{i}=l_{i} \text { for all } 1 \leq i \leq\left\lfloor\sigma_{0} \log d\right\rfloor-1 \mid \widehat{W}_{\left\lfloor\sigma_{0} \log d\right\rfloor-1} \neq \emptyset\right) .
$$

Then,

$$
\begin{aligned}
& P_{\lambda, d}\left(\sum_{i=0}^{\left\lfloor\sigma_{0} \log d\right\rfloor-1} \eta_{i} \geq\left\lfloor\frac{\sqrt{\log d}}{N(d)}\right\rfloor \mid \widehat{W}_{\left\lfloor\sigma_{0} \log d\right\rfloor-1} \neq \emptyset\right)=\sum_{\vec{l}} \gamma(\vec{l}) P_{\lambda, d}( \\
& \left.\sum_{i=0}^{\left\lfloor\sigma_{0} \log d\right\rfloor-1} \eta_{i}(\vec{l}) \geq\left\lfloor\frac{\sqrt{\log d}}{N(d)}\right\rfloor \mid X_{i}=l_{i} \text { for all } 1 \leq i \leq\left\lfloor\sigma_{0} \log d\right\rfloor-1, \widehat{W}_{\left\lfloor\sigma_{0} \log d\right\rfloor-1} \neq \emptyset\right),
\end{aligned}
$$

where

$$
\eta_{i}(\vec{l})=\sum_{j=1}^{\left\lfloor\frac{d}{N(d)}\right\rfloor} 1_{\left\{\widehat{U}_{i j}<Y\left(l_{i}\right)\right\}} .
$$

The condition $\left\{X_{i}=l_{i}\right.$ for all $\left.1 \leq i \leq\left\lfloor\sigma_{0} \log d\right\rfloor, \widehat{W}_{\left\lfloor\sigma_{0} \log d\right\rfloor} \neq \emptyset\right\}$ in Equation (4.27) is concerned with the values of $Y\left(l_{i}\right)$ and $\left\{U\left(l_{i}, l_{i}(j)\right): 1 \leq j \leq d-\left\lfloor\frac{d}{N(d)}\right\rfloor\right\}$ for 
$0 \leq i \leq\left\lfloor\sigma_{0} \log d\right\rfloor-1$. A worse condition for $\sum_{i=0}^{\left\lfloor\sigma_{0} \log d\right\rfloor-1} \eta_{i}(\vec{l}) \geq\left\lfloor\frac{\sqrt{\log d}}{N(d)}\right\rfloor$ to occur is that

$$
Y\left(l_{i}\right)<\inf \left\{U\left(l_{i}, l_{i}(j)\right): 1 \leq j \leq d-\left\lfloor\frac{d}{N(d)}\right\rfloor\right\}
$$

for all $0 \leq i \leq\left\lfloor\sigma_{0} \log d\right\rfloor-1$, i.e.,

$$
\begin{aligned}
& P_{\lambda, d}\left(\sum_{i=0}^{\left\lfloor\sigma_{0} \log d\right\rfloor-1} \eta_{i}(\vec{l}) \geq\left\lfloor\frac{\sqrt{\log d}}{N(d)}\right\rfloor \mid X_{i}=l_{i} \text { for all } 1 \leq i \leq\left\lfloor\sigma_{0} \log d\right\rfloor-1,\right. \\
& \left.\widehat{W}_{\left\lfloor\sigma_{0} \log d\right\rfloor-1} \neq \emptyset\right) \geq \\
& P_{\lambda, d}\left(\sum_{i=0}^{\left\lfloor\sigma_{0} \log d\right\rfloor-1} \eta_{i}(\vec{l}) \geq\left\lfloor\frac{\sqrt{\log d}}{N(d)}\right\rfloor \mid Y\left(l_{i}\right)<\inf \left\{U\left(l_{i}, l_{i}(j)\right): 1 \leq j \leq d-\left\lfloor\frac{d}{N(d)}\right\rfloor\right\}\right.
\end{aligned}
$$

for all $\left.0 \leq i \leq\left\lfloor\sigma_{0} \log d\right\rfloor-1\right)$.

Note that $Y\left(l_{i}\right)$ is with the same distribution as that of $\widehat{Y}_{i}$ while $\eta_{i}(\vec{l})$ is with the same distribution as that of $\xi_{i}$. Further more, inf $\left\{U\left(l_{i}, l_{i}(j)\right): 1 \leq j \leq d-\left\lfloor\frac{d}{N(d)}\right\rfloor\right\}$ is an exponential time with rate at most

$$
\left(d-\left\lfloor\frac{d}{N(d)}\right\rfloor\right) \frac{\lambda}{d} M^{2} \leq \lambda M^{2}
$$

which is the rate of $\Lambda_{i}$. Then,

$$
P_{\lambda, d}\left(\sum_{i=0}^{\left\lfloor\sigma_{0} \log d\right\rfloor-1} \eta_{i}(\vec{l}) \geq\left\lfloor\frac{\sqrt{\log d}}{N(d)}\right\rfloor \mid Y\left(l_{i}\right)<\inf \left\{U\left(l_{i}, l_{i}(j)\right): j \leq d-\left\lfloor\frac{d}{N(d)}\right\rfloor-1\right\}\right.
$$

for all $\left.1 \leq i \leq\left\lfloor\sigma_{0} \log d\right\rfloor-1\right)$

$$
\geq P_{\lambda, d}\left(\sum_{i=0}^{\left\lfloor\sigma_{0} \log d\right\rfloor-1} \xi_{i} \geq\left\lfloor\frac{\sqrt{\log d}}{N(d)}\right\rfloor \mid \widehat{Y}_{i}<\Lambda_{i} \text { for all } 0 \leq i \leq\left\lfloor\sigma_{0} \log d\right\rfloor-1\right) .
$$

Therefore, by Equation (4.28),

$$
\begin{aligned}
& P_{\lambda, d}\left(\sum_{i=0}^{\left\lfloor\sigma_{0} \log d\right\rfloor-1} \eta_{i}(\vec{l}) \geq\left\lfloor\frac{\sqrt{\log d}}{N(d)}\right\rfloor \mid X_{i}=l_{i} \text { for all } i<\left\lfloor\sigma_{0} \log d\right\rfloor, \widehat{W}_{\left\lfloor\sigma_{0} \log d\right\rfloor-1} \neq \emptyset\right) \\
& \geq P_{\lambda, d}\left(\sum_{i=0}^{\left\lfloor\sigma_{0} \log d\right\rfloor-1} \xi_{i} \geq\left\lfloor\frac{\sqrt{\log d}}{N(d)}\right\rfloor \mid \widehat{Y}_{i}<\Lambda_{i} \text { for all } 0 \leq i \leq\left\lfloor\sigma_{0} \log d\right\rfloor-1\right) .
\end{aligned}
$$

By Equations (4.27) and (4.29),

$$
\begin{aligned}
& P_{\lambda, d}\left(\sum_{i=0}^{\left\lfloor\sigma_{0} \log d\right\rfloor-1} \eta_{i} \geq\left\lfloor\frac{\sqrt{\log d}}{N(d)}\right\rfloor \mid \widehat{W}_{\left\lfloor\sigma_{0} \log d\right\rfloor-1} \neq \emptyset\right) \\
& \geq \sum_{\vec{l}} \gamma(\vec{l}) P_{\lambda, d}\left(\sum_{i=0}^{\left\lfloor\sigma_{0} \log d\right\rfloor-1} \xi_{i} \geq K(d) \mid \widehat{Y}_{i}<\Lambda_{i} \text { for all } 0 \leq i \leq\left\lfloor\sigma_{0} \log d\right\rfloor-1\right) \\
& =P_{\lambda, d}\left(\sum_{i=0}^{\left\lfloor\sigma_{0} \log d\right\rfloor-1} \xi_{i} \geq K(d) \mid \widehat{Y}_{i}<\Lambda_{i} \text { for all } 0 \leq i \leq\left\lfloor\sigma_{0} \log d\right\rfloor-1\right),
\end{aligned}
$$


since $\sum_{\vec{l}} \gamma(\vec{l})=1$. Equation (4.22) follows directly from Equations (4.25), (4.26) and $(4.30)$.

Equation (4.23) follows from the following analysis. According to the assumption of independence of the exponential times,

$$
\begin{aligned}
& E_{\lambda, d}\left(e^{-s \frac{N(d)}{\log d} \sum_{j=0}^{\left\lfloor\sigma_{0} \log d\right\rfloor-1} \xi_{j}} \mid \widehat{Y}_{i}<\Lambda_{i} \text { for all } 0 \leq i \leq\left\lfloor\sigma_{0} \log d\right\rfloor-1\right) \\
& =\left[E_{\lambda, d}\left(e^{-s \frac{N(d)}{\log d} \xi_{0}} \mid \widehat{Y}_{0}<\Lambda_{0}\right)\right]^{\left\lfloor\sigma_{0} \log d\right\rfloor} .
\end{aligned}
$$

By direct calculation,

$$
E_{\lambda, d}\left(e^{-s \frac{N(d)}{\log d} \xi_{0}} \mid \widehat{Y}_{0}<\Lambda_{0}\right)=E\left(\Xi\left(\widehat{Y}_{0}, s, d\right) \mid \widehat{Y}_{0}<\Lambda_{0}\right),
$$

where

$$
\Xi(t, s, d)=\left[E\left(e^{-s \frac{N(d)}{\log d}}\left(1-e^{-\frac{\lambda t \epsilon \rho}{d}}\right)+e^{-\frac{\lambda t \epsilon \rho}{d}}\right)\right]^{\left\lfloor\frac{d}{N(d)}\right\rfloor} .
$$

Then, by Equation (4.31),

$$
\begin{aligned}
& E_{\lambda, d}\left(e^{-s \frac{N(d)}{\log d} \sum_{j=0}^{\left\lfloor\sigma_{0} \log d\right\rfloor-1} \xi_{j}} \mid \widehat{Y}_{i}<\Lambda_{i} \text { for all } 0 \leq i \leq\left\lfloor\sigma_{0} \log d\right\rfloor-1\right) \\
& =\left[E\left(\Xi\left(\widehat{Y}_{0}, s, d\right) \mid \widehat{Y}_{0}<\Lambda_{0}\right)\right]^{\left\lfloor\sigma_{0} \log d\right\rfloor} .
\end{aligned}
$$

By direct calculation, it is not difficult to check that

$$
\lim _{d \rightarrow+\infty}\left\lfloor\sigma_{0} \log d\right\rfloor(\Xi(t, s, d)-1)=-s \sigma \lambda t \epsilon E \rho .
$$

for any $t>0$. As a result,

$$
\Theta(s)=\lim _{d \rightarrow+\infty}\left[E\left(\Xi\left(\widehat{Y}_{0}, s, d\right) \mid \widehat{Y}_{0}<\Lambda_{0}\right)\right]^{\left\lfloor\sigma_{0} \log d\right\rfloor}=E\left(e^{-s \sigma \lambda \widehat{Y}_{0} \epsilon E \rho} \mid \widehat{Y}_{0}<\Lambda_{0}\right) .
$$

Note that here we still utilize the fact that $\left(1+a_{d}\right)^{c_{d}} \rightarrow e^{c}$ when $a_{d} \rightarrow 0, c_{d} \rightarrow+\infty$ and $a_{d} c_{d} \rightarrow c$. Equation (4.23) follows directly from Equations (4.32) and (4.33).

4.4. Proof of Lemma 4.2. In this subsection we give the proof of Lemma 4.2. The proof is inspired a lot by the approach introduced in Xue (2015). First we introduce some definitions and notations. We let $\left\{\vartheta_{n}\right\}_{n \geq 0}$ be the oriented random walk on $\mathbb{Z}_{+}^{d}$ such that

$$
P\left(\vartheta_{n+1}-\vartheta_{n}=e_{i}\right)=\frac{1}{d}
$$

for each $n \geq 0$ and $1 \leq i \leq d$. We let $\left\{\nu_{n}\right\}_{n \geq 0}$ be an independent copy of $\left\{\vartheta_{n}\right\}_{n \geq 0}$. From now on, we denote by $\mathbb{P}$ the probability measure of $\left\{\vartheta_{n}\right\}_{n \geq 0}$ and $\left\{\nu_{n}\right\}_{n \geq 0}$ while denote by $\mathbb{E}$ the expectation with respect to $\mathbb{P}$. When we need to point out the dimension $d$ of the lattice, we write $\mathbb{P}$ and $\mathbb{E}$ as $\mathbb{P}_{d}$ and $\mathbb{E}_{d}$. We write $\vartheta_{n}$ (resp. 
$\left.\nu_{n}\right)$ as $\vartheta_{n}^{x}\left(\operatorname{resp} . \nu_{n}^{x}\right)$ when $\vartheta_{0}=x\left(\operatorname{resp} . \nu_{0}=x\right)$. For $x, y \in \mathbb{Z}_{+}^{d}$ satisfying $x \neq y$ and $\|x\|=\|y\|$, we define

$$
\tau_{x, y}=\inf \left\{k \geq 1: \vartheta_{k}^{x}=\nu_{k}^{y}\right\}
$$

That is to say, $\tau_{x, y}$ is the first moment when $\left\{\vartheta_{n}^{x}\right\}_{n \geq 0}$ and $\left\{\nu_{n}^{y}\right\}_{n \geq 0}$ collide. For $x, y \in \mathbb{Z}_{+}^{d}$ satisfying $\|x\|=\|y\|$, we introduce the following random variables. We define

$$
\tau_{0}^{x, y}= \begin{cases}0 & \text { if } x=y \\ \tau_{x, y} & \text { if } x \neq y\end{cases}
$$

We let

$$
\begin{aligned}
& \tau_{1}^{x, y}=\inf \left\{n \geq \tau_{0}^{x, y}: \vartheta_{n}^{x}=\nu_{n}^{y}, \vartheta_{n+1}^{x}=\nu_{n+1}^{y}\right\} \\
& \kappa_{1}^{x, y}=\inf \left\{n>\tau_{1}^{x, y}: \vartheta_{n}^{x}=\nu_{n}^{y}, \vartheta_{n+1}^{x} \neq \nu_{n+1}^{y}\right\} \\
& \tau_{2}^{x, y}=\inf \left\{n>\kappa_{1}^{x, y}: \vartheta_{n}^{x}=\nu_{n}^{y}, \vartheta_{n+1}^{x}=\nu_{n+1}^{y}\right\} \\
& \kappa_{2}^{x, y}=\inf \left\{n>\tau_{2}^{x, y}: \vartheta_{n}^{x}=\nu_{n}^{y}, \vartheta_{n+1}^{x} \neq \nu_{n+1}^{y}\right\}, \\
& \ldots \ldots \\
& \tau_{l}^{x, y}=\inf \left\{n>\kappa_{l-1}^{x, y}: \vartheta_{n}^{x}=\nu_{n}^{y}, \vartheta_{n+1}^{x}=\nu_{n+1}^{y}\right\} \\
& \kappa_{l}^{x, y}=\inf \left\{n>\tau_{l}^{x, y}: \vartheta_{n}^{x}=\nu_{n}^{y}, \vartheta_{n+1}^{x} \neq \nu_{n+1}^{y}\right\} \\
& \ldots \ldots
\end{aligned}
$$

That is to say, $\tau_{1}^{x, y}$ is the first moment $n$ such that $\vartheta_{n}^{x}=\nu_{n}^{y}$ and $\vartheta_{n+1}^{x}=\nu_{n+1}^{y}$. For $l \geq 1, \kappa_{l}^{x, y}$ is the first moment $n$ after $\tau_{l}^{x, y}$ such that $\vartheta_{n}^{x}=\nu_{n}^{y}$ and $\vartheta_{n+1}^{x} \neq \nu_{n+1}^{y}$ while $\tau_{l+1}^{x, y}$ is the first moment $n$ after $\kappa_{l}^{x, y}$ that $\vartheta_{n}^{x}=\nu_{n}^{y}$ and $\vartheta_{n+1}^{x}=\nu_{n+1}^{y}$.

We define

$$
T(x, y)= \begin{cases}\sup \left\{l \geq 0: \tau_{l}^{x, y}<+\infty\right\} & \text { if } \tau_{0}^{x, y}<+\infty \\ 0 & \text { if } \tau_{0}^{x, y}=+\infty\end{cases}
$$

In this subsection we assume that $d \geq 4$ such that $T(x, y)<+\infty$ with probability one according to the conclusion given in Cox and Durrett (1983) about the collision times of two independent oriented random walks.

For $1 \leq l \leq T(x, y)$, we define

$$
h_{l}^{x, y}=\kappa_{l}^{x, y}-\tau_{l}^{x, y} .
$$

We let

$$
\begin{aligned}
& f_{0}^{x, y}=\left|\left\{\tau_{0}^{x, y} \leq n<\tau_{1}^{x, y}: \vartheta_{n}^{x}=\nu_{n}^{y}\right\}\right|, \\
& f_{1}^{x, y}=\left|\left\{\kappa_{1}^{x, y}<n<\tau_{2}^{x, y}: \vartheta_{n}^{x}=\nu_{n}^{y}\right\}\right|, \\
& \ldots \ldots \\
& f_{l}^{x, y}=\left|\left\{\kappa_{l}^{x, y}<n<\tau_{l+1}^{x, y}: \vartheta_{n}^{x}=\nu_{n}^{y}\right\}\right|, \\
& \ldots \ldots \\
& f_{T(x, y)-1}^{x, y}=\left|\left\{\kappa_{T(x, y)-1}^{x, y}<n<\tau_{T(x, y)}^{x, y}: \vartheta_{n}^{x}=\nu_{n}^{y}\right\}\right|, \\
& f_{T(x, y)}^{x, y}=\left|\left\{n>\kappa_{T(x, y)}^{x, y}: \vartheta_{n}^{x}=\nu_{n}^{y}\right\}\right|,
\end{aligned}
$$


where $|\mathcal{A}|$ is the cardinality of the set $\mathcal{A}$ as we have introduced. Then, for $x, y$ that $\|x\|=\|y\|$, we define

$$
\begin{aligned}
& R(x, y)= \\
& \frac{2^{T(x, y)+\sum_{i=0}^{T(x, y)} f_{i}^{x, y}}\left(1+\frac{\lambda M^{2}}{d}\right)}{4 T(x, y)+2 \sum_{i=1}^{T(x, y)} h_{i}^{x, y}+4 \sum_{i=0}^{T(x, y)} f_{i}^{x, y} M^{6 T(x, y)+4} \sum_{i=0}^{T(x, y)} f_{i}^{x, y}} \\
& \left(\frac{\lambda E\left(\rho^{2}\right)}{d}\right)^{T(x, y)} h_{i}^{x, y}\left(E\left(\rho^{2}\right)\right)^{3 T(x, y)+2} \sum_{i=0}^{T(x, y)} f_{i}(x, y)
\end{aligned}
$$

when $\tau_{0}^{x, y}>0$ while define

$$
\begin{aligned}
& R(x, y)=
\end{aligned}
$$

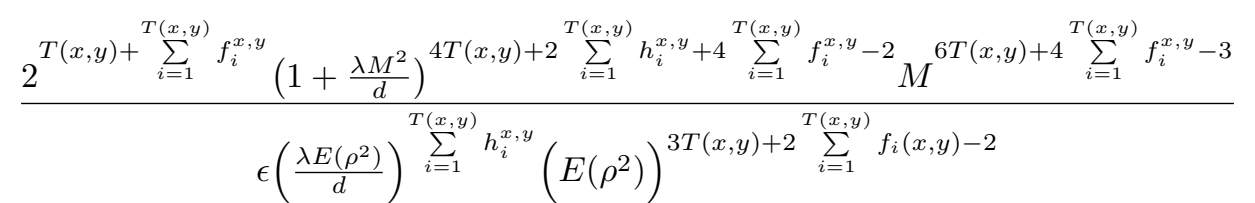

when $\tau_{0}^{x, y}=\tau_{1}^{x, y}=0$ and define

$$
\begin{aligned}
& R(x, y)= \\
& \frac{2^{T(x, y)+\sum_{i=0}^{T(x, y)} f_{i}^{x, y}}\left(1+\frac{\lambda M^{2}}{d}\right)^{4 T(x, y)+2} \sum_{i=1}^{T(x, y)} h_{i}^{x, y}+4 \sum_{i=0}^{T(x, y)} f_{i}^{x, y}-2 M^{6 T(x, y)+4} \sum_{i=0}^{T(x, y)} f_{i}^{x, y}-4}{\left(\frac{\lambda E\left(\rho^{2}\right)}{d}\right) \sum_{i=1}^{T(x)} h_{i}^{x, y}\left(E\left(\rho^{2}\right)\right)^{3 T(x, y)+2} \sum_{i=0}^{T(x, y)} f_{i}(x, y)-2}
\end{aligned}
$$

when $0=\tau_{0}^{x, y}<\tau_{1}^{x, y}$. The following three lemmas are crucial for us to prove Lemma 4.2.

Lemma 4.6. There exists $c_{1}>0$ which does not depend on $d$ such that

$$
\mathbb{P}_{d}\left(\tau_{x, y}<+\infty\right) \leq \frac{c_{1}}{d^{2}}
$$

for any $d \geq 4$ and $x, y \in \mathbb{Z}_{+}^{d}$ satisfying $x \neq y,\|x\|=\|y\|$.

Lemma 4.7. For given $\lambda>\frac{1}{E\left(\rho^{2}\right)}$, there exist $d_{0} \geq 4$ and $c_{2}>0$ which does not depend on d such that

$$
\mathbb{E}_{d}(R(x, y) \mid \tau(x, y)<+\infty) \leq c_{2}
$$

for any $d \geq d_{0}$ and $x, y \in \mathbb{Z}_{d}^{+}$satisfying $\|x\|=\|y\|$.

Lemma 4.8. For $A \subseteq \mathbb{Z}_{d}^{+}$satisfying $\|x\|=\|y\|$ for any $x, y \in A$,

$$
P_{\lambda, d}\left(I_{t}^{A} \neq \emptyset, \forall t \geq 0 \mid \rho(x)=\epsilon \text { for all } x \in A\right) \geq \frac{1}{\frac{1}{|A|^{2}} \sum_{x \in A} \sum_{y \in A} \mathbb{E}_{d}(R(x, y))} .
$$

The proofs of Lemmas 4.6-4.8 will be given later. Now we give the proof of Lemma 4.2.

Proof of Lemma 4.2: For $x, y \in \mathbb{Z}_{+}^{d}$ satisfying $x \neq y,\|x\|=\|y\|$, according to the definition of $R(x, y), R(x, y)=1$ when $\tau_{x, y}=+\infty$. Therefore, by Lemmas 4.6 and 4.7 ,

$$
\mathbb{E}_{d}(R(x, y))=\mathbb{P}_{d}\left(\tau_{x, y}=+\infty\right)+\mathbb{E}_{d}\left(R(x, y) 1_{\left\{\tau_{x, y}<+\infty\right\}}\right) \leq 1+\frac{c_{1} c_{2}}{d^{2}}
$$


for any $d \geq d_{0}$ and $x, y \in \mathbb{Z}_{+}^{d}$ satisfying $x \neq y,\|x\|=\|y\|$. By Lemma 4.7 and Equation (4.34),

$$
\sum_{x \in A} \sum_{y \in A} \mathbb{E}_{d}(R(x, y)) \leq|A| c_{2}+\left(|A|^{2}-|A|\right)\left(1+\frac{c_{1} c_{2}}{d^{2}}\right)
$$

for any $A \subseteq \mathbb{Z}_{+}^{d}$ satisfying $\|x\|=\|y\|$ for any $x, y \in A$. By Lemma 4.8 and Equation (4.35),

$$
P_{\lambda, d}\left(I_{t}^{A} \neq \emptyset, \forall t \geq 0 \mid \rho(x)=\epsilon \text { for all } x \in A\right) \geq \frac{|A|^{2}}{\left(|A|^{2}-|A|\right)\left(1+\frac{c_{1} c_{2}}{d^{2}}\right)+|A| c_{2}}
$$

for any $d \geq d_{0}$ and $A \subseteq \mathbb{Z}_{+}^{d}$ satisfying $\|x\|=\|y\|$ for any $x, y \in A$. Note that $\|x\|=$ $\left\lfloor\sigma_{0} \log d\right\rfloor$ for any $x \in \Gamma_{1} \bigcap \Gamma_{2}$. Hence, let $m(d)=\frac{|K(d)|^{2}}{\left(|K(d)|^{2}-|K(d)|\right)\left(1+\frac{c_{1} c_{2}}{d^{2}}\right)+|K(d)| c_{2}}$, then $\lim _{d \rightarrow+\infty} m(d)=1$ while

$$
P_{\lambda, d}\left(I_{t}^{A} \neq \emptyset, \forall t \geq 0 \mid \rho(x)=\epsilon \text { for all } x \in A\right) \geq m(d)
$$

for any $A$ satisfying $A \subseteq \Gamma_{1} \cap \Gamma_{2},|A|=K(d)$ by Equation (4.36) and the proof is complete.

Now we give the proof of Lemma 4.6.

Proof of Lemma 4.6: Let

$$
\tau_{O, O}=\inf \left\{n \geq 1: \vartheta_{n}^{O}=\nu_{n}^{O}\right\},
$$

then by the conclusion given in Cox and Durrett (1983), there exists $c_{3}>0$ which does not depend on $d$ such that

$$
\mathbb{P}_{d}\left(\tau_{O, O}<+\infty\right) \leq \frac{1}{d}+\frac{c_{3}}{d^{2}}
$$

for all $d \geq 4$. Since $\mathbb{P}_{d}\left(\tau_{O, O}=1\right)=\frac{1}{d}$, according to the spatial homogeneity of $\mathbb{Z}_{+}^{d}$,

$$
\left(1-\frac{1}{d}\right) \mathbb{P}_{d}\left(\tau_{e_{i}, e_{j}}<+\infty\right)=\mathbb{P}_{d}\left(2 \leq \tau_{O, O}<+\infty\right) \leq \frac{c_{3}}{d^{2}}
$$

for any $d \geq 4,1 \leq i<j \leq d$. For $x, y$ that $x \neq y$ and $\|x\|=\|y\|,\|x-y\|$ is an even number, which is at least two. Let

$$
\widehat{\tau}_{x, y}=\inf \left\{n \geq 0:\left\|\vartheta_{n}^{x}-\nu_{n}^{y}\right\|=2\right\},
$$

then, according to the strong Markov property,

$$
\mathbb{P}_{d}\left(\tau_{x, y}<+\infty\right)=\mathbb{P}_{d}\left(\widehat{\tau}_{x, y}<+\infty\right) \mathbb{P}_{d}\left(\tau_{e_{1}, e_{2}}<+\infty\right) \leq \frac{c_{3}}{d(d-1)},
$$

since $\left\|\vartheta_{n+1}^{x}-\nu_{n+1}^{y}\right\|-\left\|\vartheta_{n}^{x}-\nu_{n}^{y}\right\| \in\{0,-2,2\}$ for each $n$.

For $d \geq 4$,

$$
\frac{c_{3}}{d(d-1)} \leq \frac{2 c_{3}}{d^{2}}
$$

Let $c_{1}=2 c_{3}$ and the proof is complete.

Now we give the proof of Lemma 4.7. 
Proof of Lemma 4.7: According to the definition of $R(\cdot, \cdot)$, for any $x, y \in \mathbb{Z}_{d}^{+}$,

$$
\begin{aligned}
& R(x, y) \leq \\
& \frac{c_{5} 2^{T(x, y)+\sum_{i=0}^{T(x, y)} f_{i}^{x, y}}\left(1+\frac{\lambda M^{2}}{d}\right)^{4 T(x, y)+2} \sum_{i=1}^{T(x, y)} h_{i}^{x, y}+4 \sum_{i=0}^{T(x, y)} f_{i}^{x, y} M^{6 T(x, y)+4} \sum_{i=0}^{T(x, y)} f_{i}^{x, y}}{\left(\frac{\lambda E\left(\rho^{2}\right)}{d}\right)^{\left.\sum_{i=1}, y\right)} h_{i}^{x, y}\left(E\left(\rho^{2}\right)\right)^{3 T(x, y)+2} \sum_{i=0}^{T(x, y)} f_{i}(x, y)},
\end{aligned}
$$

where $c_{5}>0$ is a constant which depends on $M, E\left(\rho^{2}\right), E \rho, \lambda, \epsilon$ and does not depend on $d$. According to the strong Markov property, for any positive integers $T,\left\{h_{i}\right\}_{i=1}^{T},\left\{f_{i}\right\}_{i=0}^{T}$,

$$
\begin{aligned}
& \mathbb{P}_{d}\left(T(x, y)=T, h_{i}^{x, y}=h_{i}, f_{i}^{x, y}=f_{i} \text { for } 0 \leq i \leq T \mid \tau_{x, y}<+\infty\right) \\
& \leq \mathbb{P}_{d}\left(2 \leq \tau_{O, O}<+\infty\right)^{f_{0}+\sum_{i=1}^{T-1}\left(f_{i}+1\right)+f_{T}} \mathbb{P}_{d}\left(\tau_{O, O}=1\right)^{\sum_{i=1}^{T} h_{i}},
\end{aligned}
$$

where $\tau_{O, O}$ is defined as in the proof of Lemma 4.6. By Equations (4.38) and (4.41),

$$
\begin{aligned}
& \mathbb{P}_{d}\left(T(x, y)=T, h_{i}^{x, y}=h_{i}, f_{i}^{x, y}=f_{i} \text { for } 0 \leq i \leq T \mid \tau_{x, y}<+\infty\right) \\
& \leq\left(\frac{c_{3}}{d^{2}}\right)^{f_{0}-1+\sum_{i=1}^{T} f_{i}+T}\left(\frac{1}{d}\right)^{\sum_{i=1}^{T} h_{i}} .
\end{aligned}
$$

By Equations (4.40) and (4.42),

$$
\begin{aligned}
& \mathbb{E}_{d}\left(R(x, y) \mid \tau_{x, y}<+\infty\right) \\
& \leq c_{5} \sum_{T=0}^{+\infty} \sum_{f_{0}=1}^{+\infty} \sum_{f_{1}=1}^{+\infty} \ldots \sum_{f_{T}=1}^{+\infty} \sum_{h_{1}=1}^{+\infty} \ldots \sum_{h_{T}=1}^{+\infty}\left(\frac{c_{3}}{d^{2}}\right)^{\sum_{i=0}^{T} f_{i}+T-1}\left(\frac{1}{d}\right)^{\sum_{i=1}^{T} h_{i}}
\end{aligned}
$$

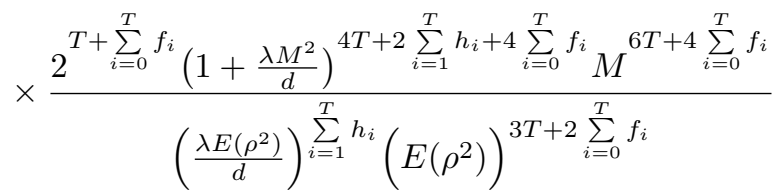

$$
\begin{aligned}
& =\widehat{c}_{5} \sum_{T=0}^{+\infty}\left(c_{7}(d)\right)^{T}\left[\sum_{f_{0}=0}^{+\infty} \sum_{f_{1}=1}^{+\infty} \ldots \sum_{f_{T}=1}^{+\infty}\left(c_{8}(d)\right)^{\sum_{i=0}^{T} f_{i}}\right] \\
& \times\left[\sum_{h_{1}=1}^{+\infty} \ldots \sum_{h_{T}=1}^{+\infty}\left(c_{9}(d)\right)^{\sum_{i=1}^{T} h_{i}}\right]
\end{aligned}
$$

where $\widehat{c}_{5}=\frac{2 c_{5}\left(1+\frac{\lambda M^{2}}{d}\right)^{4} M^{4}}{\left(E\left(\rho^{2}\right)\right)^{2}}, c_{7}(d)=\frac{2 c_{3}\left(1+\frac{\lambda M^{2}}{d}\right)^{4} M^{6}}{d^{2}\left(E\left(\rho^{2}\right)\right)^{3}}, c_{8}(d)=\frac{2 c_{3}\left(1+\frac{\lambda M^{2}}{d}\right)^{4} M^{4}}{d^{2}\left(E\left(\rho^{2}\right)\right)^{2}}$ and

$$
c_{9}(d)=\frac{1}{d} \frac{d}{\lambda E\left(\rho^{2}\right)}\left(1+\frac{\lambda M^{2}}{d}\right)^{2}=\frac{\left(1+\frac{\lambda M^{2}}{d}\right)^{2}}{\lambda E\left(\rho^{2}\right)} .
$$

Since $\lambda>\frac{1}{E\left(\rho^{2}\right)}$, there exists $c_{10} \in(0,1)$, which does not depend on $d$, such that

$$
\widehat{c}_{5}<\frac{3 M^{4} c_{5}}{\left(E\left(\rho^{2}\right)\right)^{2}} \text { and } c_{9}(d) \leq c_{10}
$$


for sufficiently large $d$. For sufficiently large $d$,

$$
c_{8}(d) \leq \frac{1}{2} \text { and } \frac{c_{7}(d) c_{8}(d)}{1-c_{8}(d)} \frac{c_{10}}{1-c_{10}} \leq \frac{1}{10}
$$

since $\lim _{d \rightarrow+\infty} c_{7}(d)=\lim _{d \rightarrow+\infty} c_{8}(d)=0$. As a result, by Equation (4.43),

$$
\begin{aligned}
& \mathbb{E}_{d}\left(R(x, y) \mid \tau_{x, y}<+\infty\right) \\
& \leq \frac{1}{1-c_{8}(d)} \frac{3 M^{4} c_{5}}{\left(E\left(\rho^{2}\right)\right)^{2}} \sum_{T=0}^{+\infty}\left(c_{7}(d) \frac{c_{8}(d)}{1-c_{8}(d)} \frac{c_{10}}{1-c_{10}}\right)^{T} \\
& \leq \frac{6 M^{4} c_{5}}{\left(E\left(\rho^{2}\right)\right)^{2}} \sum_{T=0}^{+\infty}\left(\frac{1}{10}\right)^{T}=\frac{20 M^{4} c_{5}}{3\left(E\left(\rho^{2}\right)\right)^{2}}
\end{aligned}
$$

for sufficiently large $d$. Let

$$
c_{2}=\frac{20 M^{4} c_{5}}{3\left(E\left(\rho^{2}\right)\right)^{2}}
$$

and the proof is complete.

At the end of this subsection, we give the proof of Lemma 4.8 .

Proof of Lemma 4.8: For given $A \subseteq \mathbb{Z}_{+}^{d}$ satisfying $\|x\|=\|y\|$ for any $x, y \in A$, we use $\widetilde{P}_{\lambda, d}(\cdot)$ to denote the conditional probability measure

$$
P_{\lambda, d}(\cdot \mid \rho(x)=\epsilon \text { for all } x \in A) .
$$

We use $\widetilde{E}_{\lambda, d}$ to denote the expectation with respect to $\widetilde{P}_{\lambda, d}$.

For each $m \geq 1$ and each $x \in A$, we define

$$
L_{m}(x)=\left\{\vec{x}=\left(x_{0}, x_{1}, \ldots, x_{m}\right): x_{0}=x, x_{i} \rightarrow x_{i+1} \text { for all } 0 \leq i \leq m-1\right\}
$$

as the set of oriented paths starting at $x$ with length $m$.

For each $\vec{x}=\left(x, x_{1}, \ldots, x_{m}\right) \in L_{m}(x)$, we denote by $\pi_{\vec{x}}$ the event that

$$
\widetilde{U}\left(x_{i}, x_{i+1}\right)<\tilde{Y}\left(x_{i}\right)
$$

for all $0 \leq i \leq m-1$, then for each $x \in \mathbb{Z}_{d}^{+}$,

$$
\begin{aligned}
\widetilde{P}_{\lambda, d}\left(\pi_{\vec{x}}\right) & =E_{\mu_{d}}\left(\prod_{i=0}^{m-1} P_{\lambda, \omega}\left(\widetilde{U}\left(x_{i}, x_{i+1}\right)<\widetilde{Y}\left(x_{i}\right)\right) \mid \rho(x)=\epsilon\right) \\
& =E_{\mu_{d}}\left(\frac{\frac{\lambda}{d} \epsilon \rho\left(x_{1}\right)}{1+\frac{\lambda}{d} \epsilon \rho\left(x_{1}\right)} \prod_{i=1}^{m-1} \frac{\frac{\lambda}{d} \rho\left(x_{i}\right) \rho\left(x_{i+1}\right)}{1+\frac{\lambda}{d} \rho\left(x_{i}\right) \rho\left(x_{i+1}\right)}\right) \\
& =E\left(\frac{\frac{\lambda}{d} \epsilon \rho_{1}}{1+\frac{\lambda}{d} \epsilon \rho_{1}} \prod_{i=0}^{m-1} \frac{\frac{\lambda}{d} \rho_{i} \rho_{i+1}}{1+\frac{\lambda}{d} \rho_{i} \rho_{i+1}}\right)
\end{aligned}
$$

where $\rho_{1}, \ldots, \rho_{m}$ are independent copies of $\rho$. For $x, y \in A$ and $\vec{x}=\left(x, x_{1}, \ldots, x_{m}\right)$ $\in L_{m}(x), \vec{y}=\left(y, y_{1}, \ldots, y_{m}\right) \in L_{m}(y)$,

$$
\widetilde{P}_{\lambda, d}\left(\pi_{\vec{x}} \bigcap \pi_{\vec{y}}\right)=E_{\lambda, d}\left(\prod_{l=1}^{m} G\left(x_{l-1}, y_{l-1} ; x_{l}, y_{l}\right) \mid \rho(x)=\rho(y)=\epsilon\right),
$$


where

$$
G(x, y ; u, v)=P_{\lambda, \omega}(\widetilde{U}(x, u)<\tilde{Y}(x), \widetilde{U}(y, v)<\tilde{Y}(y))
$$

for $x \rightarrow u$ and $y \rightarrow v$.

By direct calculation, for $x, y$ that $\|x\|=\|y\|$,

$$
G(x, y ; u, v) \begin{cases}=\frac{\frac{\lambda}{d} \rho(x) \rho(u)}{1+\frac{\lambda}{d} \rho(x) \rho(u)} & \text { if } x=y \text { and } u=v, \\ \leq \frac{2 \frac{\lambda^{2}}{d^{2}} \rho^{2}(x) \rho(u) \rho(v)}{\left[1+\frac{\lambda}{d} \rho(x) \rho(u)\right]\left[1+\frac{\lambda}{d} \rho(x) \rho(v)\right]} & \text { if } x=y \text { and } u \neq v \\ =\frac{\frac{\lambda^{2}}{d^{2}} \rho(x) \rho(y) \rho^{2}(u)}{\left[1+\frac{\lambda}{d} \rho(x) \rho(u)\right]\left[1+\frac{\lambda}{d} \rho(y) \rho(u)\right]} & \text { if } x \neq y \text { and } u=v \\ =\frac{\frac{\lambda^{2}}{d^{2}} \rho(x) \rho(y) \rho(u) \rho(v)}{\left[1+\frac{\lambda}{d} \rho(x) \rho(u)\right]\left[1+\frac{\lambda}{d} \rho(y) \rho(v)\right]} & \text { if } x \neq y \text { and } u \neq v\end{cases}
$$

According to the definition of the SIR model, for $x \in A$, if $\pi_{\vec{x}}$ occurs for some $\vec{x}=\left(x, x_{1}, \ldots, x_{m}\right) \in L_{m}(x)$, then

$$
x_{m} \in \bigcup_{t \geq 0} I_{t}^{A}
$$

As a result, on the event $\bigcap_{m=1}^{+\infty} \bigcup_{x \in A} \bigcup_{\vec{x} \in L_{m}(x)} \pi_{\vec{x}}$, there are infinite many vertices which have ever been infected and hence

$$
\widetilde{P}_{\lambda, d}\left(I_{t}^{A} \neq \emptyset, \forall t \geq 0\right) \geq \widetilde{P}_{\lambda, d}\left(\bigcap_{m=1}^{+\infty} \bigcup_{x \in A} \bigcup_{\vec{x} \in L_{m}(x)} \pi_{\vec{x}}\right)
$$

We use $\chi_{\vec{x}}$ to denote the indicator function of $\pi_{\vec{x}}$, then by the Cauchy-Schwartz's inequality and the dominated convergence theorem,

$$
\begin{aligned}
\widetilde{P}_{\lambda, d}\left(\bigcap_{m=1}^{+\infty} \bigcup_{x \in A} \bigcup_{\vec{x} \in L_{m}(x)} \pi_{\vec{x}}\right) & \geq \lim _{m \geq 1} \widetilde{P}_{\lambda, d}\left(\bigcup_{x \in A} \bigcup_{\vec{x} \in L_{m}(x)} \pi_{\vec{x}}\right) \\
& =\lim _{m \geq 1} \widetilde{P}_{\lambda, d}\left(\sum_{x \in A} \sum_{\vec{x} \in L_{m}(x)} \chi_{\vec{x}}>0\right) \\
& \geq \limsup _{m \rightarrow+\infty} \frac{\left[\widetilde{E}_{\lambda, d}\left(\sum_{x \in A} \sum_{\vec{x} \in L_{m}(x)} \chi_{\vec{x}}\right)\right]^{2}}{\widetilde{E}_{\lambda, d}\left[\left(\sum_{x \in A} \sum_{\vec{x} \in L_{m}(x)} \chi_{\vec{x}}\right)^{2}\right]} \\
& =\limsup _{m \rightarrow+\infty} \frac{\left.\sum_{x \in A} \sum_{y \in A} \sum_{x \in A} \sum_{\vec{x} \in L_{m}(x)} \sum_{\vec{y} \in L_{m}(y)} \widetilde{P}_{\lambda, d}\left(\pi_{\vec{x}}\right)\right]^{2}}{\widetilde{P}_{\lambda, d}\left(\pi_{\vec{x}} \bigcap \pi_{\vec{y}}\right)}
\end{aligned}
$$


By Equation (4.44), for given $m \geq 1$ and $\vec{x} \in L_{m}(x), \widetilde{P}_{\lambda, d}\left(\pi_{\vec{x}}\right)$ does not depend on the choice of $\vec{x}$ and $x$. Therefore, according to the fact that $\left|L_{m}(x)\right|=d^{m}$,

$$
\begin{aligned}
& \widetilde{P}_{\lambda, d}\left(\bigcap_{m=1}^{+\infty} \bigcup_{x \in A} \bigcup_{\vec{x} \in L_{m}(x)} \pi_{\vec{x}}\right) \\
& \geq \frac{1}{\liminf _{m \rightarrow+\infty} \frac{1}{|A|^{2}} \sum_{x \in A} \sum_{y \in A} \sum_{\vec{x} \in L_{m}(x)} \sum_{\vec{y} \in L_{m}(y)} \frac{1}{d^{2 m}} \frac{\widetilde{P}_{\lambda, d}\left(\pi_{\vec{x}} \bigcap \pi_{\vec{y}}\right)}{\widetilde{P}_{\lambda, d}\left(\pi_{\vec{x}}\right) \widetilde{P}_{\lambda, d}\left(\pi_{\vec{y}}\right)}} .
\end{aligned}
$$

We use $\vec{\vartheta}_{m}^{x}$ to denote the random path $\left(\vartheta_{0}^{x}, \ldots, \vartheta_{m}^{x}\right)$ while use $\vec{\nu}_{m}^{y}$ to denote the path $\left(\nu_{0}^{y}, \ldots, \nu_{m}^{y}\right)$, then by Equation (4.48),

$$
\widetilde{P}_{\lambda, d}\left(\bigcap_{m=1}^{+\infty} \bigcup_{x \in A} \bigcup_{\vec{x} \in L_{m}(x)} \pi_{\vec{x}}\right) \geq \frac{1}{\liminf _{m \rightarrow+\infty} \frac{1}{|A|^{2}} \sum_{x \in A} \sum_{y \in A} \mathbb{E}_{d}\left(\frac{\widetilde{P}_{\lambda, d}\left(\pi_{\vec{\vartheta}_{m}^{x}} \cap \pi_{\vec{\nu}_{m}^{y}}\right)}{\widetilde{P}_{\lambda, d}\left(\pi_{\vec{\vartheta}_{m}^{x}}\right) \widetilde{P}_{\lambda, d}\left(\pi_{\vec{\nu}_{m}^{y}}\right)}\right)}
$$

We bound $\frac{\widetilde{P}_{\lambda, d}\left(\pi_{\vec{\vartheta}_{m}^{x}} \cap \pi_{\vec{\nu}_{m}^{y}}\right)}{\widetilde{P}_{\lambda, d}\left(\pi_{\vec{\vartheta}_{m}^{x}}\right) \widetilde{P}_{\lambda, d}\left(\pi_{\vec{\nu}_{m}^{y}}\right)}$ from above according to the following procedure.

For the denominator

$$
\begin{aligned}
& \widetilde{P}_{\lambda, d}\left(\pi_{\vec{\vartheta}_{m}^{x}}\right) \widetilde{P}_{\lambda, d}\left(\pi_{\vec{\nu}_{m}^{y}}\right)= \\
& E_{\mu_{d}}\left(\prod_{i=0}^{m-1} \frac{\frac{\lambda}{d} \rho\left(\vartheta_{i}\right) \rho\left(\vartheta_{i+1}\right)}{1+\frac{\lambda}{d} \rho\left(\vartheta_{i}\right) \rho\left(\vartheta_{i+1}\right)} \mid \rho\left(\vartheta_{0}\right)=\epsilon\right) E_{\mu_{d}}\left(\prod_{i=0}^{m-1} \frac{\frac{\lambda}{d} \rho\left(\nu_{i}\right) \rho\left(\nu_{i+1}\right)}{1+\frac{\lambda}{d} \rho\left(\nu_{i}\right) \rho\left(\nu_{i+1}\right)} \mid \rho\left(\nu_{0}\right)=\epsilon\right),
\end{aligned}
$$

if $l \geq 1$ satisfies that $\vartheta_{l}^{x}=\nu_{l}^{y}$, then

$$
\left\{\begin{array}{c}
\frac{\frac{\lambda}{d} \rho\left(\vartheta_{l}^{x}\right) \rho\left(\vartheta_{l+1}^{x}\right)}{1+\frac{\lambda}{d} \rho\left(\vartheta_{l}^{x}\right) \rho\left(\vartheta_{l+1}^{x}\right)} \geq \frac{\frac{\lambda}{d} \rho\left(\vartheta_{l}^{x}\right) \rho\left(\vartheta_{l+1}^{x}\right)}{1+\frac{\lambda}{d} M^{2}} \\
\frac{\frac{\lambda}{d} \rho\left(\nu_{l}^{y}\right) \rho\left(\nu_{l+1}^{y}\right)}{1+\frac{\lambda}{d} \rho\left(\nu_{l}^{y}\right) \rho\left(\nu_{l+1}^{y}\right)} \geq \frac{\frac{\lambda}{d} \rho\left(\nu_{l}^{y}\right) \rho\left(\nu_{l+1}^{y}\right)}{1+\frac{\lambda}{d} M^{2}} \\
\frac{\frac{\lambda}{d} \rho\left(\vartheta_{l-1}^{x}\right) \rho\left(\vartheta_{l}^{x}\right)}{1+\frac{\lambda}{d} \rho\left(\vartheta_{l-1}^{x}\right) \rho\left(\vartheta_{l}^{x}\right)} \geq \frac{\frac{\lambda}{d} \rho\left(\vartheta_{l-1}^{x}\right) \rho\left(\vartheta_{l}^{x}\right)}{1+\frac{\lambda}{d} M^{2}} \\
\frac{\frac{\lambda}{d} \rho\left(\nu_{l-1}^{y}\right) \rho\left(\nu_{l}^{y}\right)}{1+\frac{\lambda}{d} \rho\left(\nu_{l-1}^{y}\right) \rho\left(\nu_{l}^{y}\right)} \geq \frac{\frac{\lambda}{d} \rho\left(\nu_{l-1}^{y}\right) \rho\left(\nu_{l}\right)}{1+\frac{\lambda}{d} M^{2}}
\end{array}\right.
$$

For $l=0$,

$$
\frac{\frac{\lambda}{d} \rho\left(\vartheta_{0}^{x}\right) \rho\left(\vartheta_{1}^{x}\right)}{1+\frac{\lambda}{d} \rho\left(\vartheta_{0}^{x}\right) \rho\left(\vartheta_{1}^{x}\right)} \geq \frac{\frac{\lambda}{d} \epsilon \rho\left(\vartheta_{1}^{x}\right)}{1+\frac{\lambda}{d} M^{2}} \quad \text { and } \quad \frac{\frac{\lambda}{d} \rho\left(\nu_{0}^{x}\right) \rho\left(\nu_{1}^{x}\right)}{1+\frac{\lambda}{d} \rho\left(\nu_{0}^{x}\right) \rho\left(\nu_{1}^{x}\right)} \geq \frac{\frac{\lambda}{d} \epsilon \rho\left(\nu_{1}^{x}\right)}{1+\frac{\lambda}{d} M^{2}} .
$$

For the numerator $\widetilde{P}_{\lambda, d}\left(\pi_{\vec{v}_{m}^{x}} \cap \pi_{\vec{\nu}_{m}^{y}}\right)$ with expression given by Equation (4.45),

$$
\left\{\begin{array}{l}
G\left(\vartheta_{l}^{x}, \nu_{l}^{y} ; \vartheta_{l+1}^{x}, \nu_{l+1}^{y}\right) \leq \frac{\lambda}{d} \rho\left(\vartheta_{l}^{x}\right) \rho\left(\vartheta_{l+1}^{x}\right) \\
\text { if } \vartheta_{l}^{x}=\nu_{l}^{y} \text { and } \vartheta_{l+1}^{x}=\nu_{l+1}^{y}, \\
G\left(\vartheta_{l}^{x}, \nu_{l}^{y} ; \vartheta_{l+1}^{x}, \nu_{l+1}^{y}\right) \leq \frac{2 \lambda^{2}}{d^{2}} \rho^{2}\left(\vartheta_{l}^{x}\right) \rho\left(\vartheta_{l+1}^{x}\right) \rho\left(\nu_{l+1}^{y}\right) \\
\text { if } \vartheta_{l}^{x}=\nu_{l}^{y} \text { and } \vartheta_{l+1}^{x} \neq \nu_{l+1}^{y} \\
G\left(\vartheta_{l}^{x}, \nu_{l}^{y} ; \vartheta_{l+1}^{x}, \nu_{l+1}^{y}\right) \leq \frac{\lambda^{2}}{d^{2}} \rho\left(\vartheta_{l}^{x}\right) \rho\left(\nu_{l}^{y}\right) \rho^{2}\left(\vartheta_{l+1}^{x}\right) \\
\text { if } \vartheta_{l}^{x} \neq \nu_{l}^{y} \text { and } \vartheta_{l+1}^{x}=\nu_{l+1}^{y} .
\end{array}\right.
$$

According to the aforesaid inequalities, $\frac{\widetilde{P}_{\lambda, d}\left(\pi_{\vec{\vartheta}_{m}^{x}} \cap \pi_{\vec{\nu}_{m}^{y}}\right)}{\widetilde{P}_{\lambda, d}\left(\pi_{\vec{\vartheta}_{m}^{x}}\right) \widetilde{P}_{\lambda, d}\left(\pi_{\vec{\nu}_{m}^{y}}\right)}$ is bounded from above by an upper bound $R_{m}(x, y)$. According to our assumption of the independence 
between the exponential times, the expression of $R_{m}(x, y)$ can be simplified by canceling common factors in the numerator and denominator. For example, if $l<k$ that $\vartheta_{l}^{x}=\nu_{l}^{y}$ and $\vartheta_{k}^{x}=\nu_{k}^{y}$ while $\vartheta_{j}^{x} \neq \nu_{j}^{y}$ for any $l<j<k$, then both the numerator and denominator have the factor

$$
\left(E\left(\frac{\prod_{i=1}^{k-l-1} \rho_{i}^{2}}{\prod_{i=1}^{k-l-2}\left(1+\frac{\lambda}{d} \rho_{i} \rho_{i+1}\right)}\right)\right)^{2}
$$

that can be canceled, where $\rho_{1}, \ldots, \rho_{k-1-l}$ are independent copies of $\rho$. As a result, it is not difficult to check that

$$
\lim _{m \rightarrow+\infty} R_{m}(x, y)=R(x, y)
$$

and hence

$$
\begin{aligned}
\widetilde{P}_{\lambda, d}\left(\bigcap_{m=1}^{+\infty} \bigcup_{x \in A} \bigcup_{\vec{x} \in L_{m}(x)} \pi_{\vec{x}}\right) & \geq \frac{1}{\lim _{m \rightarrow+\infty} \frac{1}{|A|^{2}} \sum_{x \in A} \sum_{y \in A} \mathbb{E}_{d}\left(R_{m}(x, y)\right)} \\
& =\frac{1}{\frac{1}{|A|^{2}} \sum_{x \in A} \sum_{y \in A} \mathbb{E}_{d}(R(x, y))}
\end{aligned}
$$

according to Equation (4.49). Lemma 4.8 follows directly from Equations (4.47) and (4.50).

\section{Proof of Equation (2.4)}

In this section we give the proof of Equation (2.4). We still assume that the vertex weight $\rho$ satisfies (4.1). The assumption is without loss of generality according to the following analysis. For general $\rho$ not satisfying (4.1), we let

$$
\widehat{\rho}_{m}= \begin{cases}\rho & \text { if } \rho \geq \frac{1}{m} \\ \frac{1}{m} & \text { if } \rho<\frac{1}{m}\end{cases}
$$

then $\widehat{\rho}_{m} \geq \rho$ and $\lim _{m \rightarrow+\infty} \widehat{\rho}_{m}=\rho$. Therefore,

$$
P_{\lambda, d, \rho}\left(C_{t}^{O} \neq \emptyset, \forall t \geq 0\right) \leq P_{\lambda, d, \widehat{\rho}_{m}}\left(C_{t}^{O} \neq \emptyset, \forall t \geq 0\right) .
$$

If Equation (2.4) holds under assumption (4.1), which $\widehat{\rho}_{m}$ satisfies, then

$$
\begin{aligned}
& \limsup _{d \rightarrow+\infty} P_{\lambda, d, \rho}\left(C_{t}^{O} \neq \emptyset, \forall t \geq 0\right) \\
& \leq \limsup _{d \rightarrow+\infty} P_{\lambda, d, \widehat{\rho}_{m}}\left(C_{t}^{O} \neq \emptyset, \forall t \geq 0\right) \leq E\left(\frac{\lambda \widehat{\rho}_{m} \widehat{\theta}_{m}}{1+\lambda \widehat{\rho}_{m} \widehat{\theta}_{m}}\right),
\end{aligned}
$$

where $\widehat{\theta}_{m}$ satisfies

$$
E\left(\frac{\lambda \widehat{\rho}_{m}^{2}}{1+\lambda \widehat{\rho}_{m} \widehat{\theta}_{m}}\right)=1
$$

and it is easy to check that $\lim _{m \rightarrow+\infty} \widehat{\theta}_{m}=\theta$. Let $m \rightarrow+\infty$, then Equation (2.4) holds for general $\rho$.

For each $n \geq 0$, we define

$$
\beta_{n}=\left\{x \in \mathbb{Z}_{+}^{d}:\|x\|=n \text { and } x \in \bigcup_{t \geq 0} C_{t}^{O}\right\}
$$


as the vertices with $l_{1}$ norm $n$ which have ever been infected in the contact process with $O$ as the unique initially infected vertex.

The infection never dies out when and only when there are infinitely many vertices that have ever been infected. Furthermore, since $x$ infects $y$ only if $x \rightarrow y$,

$$
\left\{C_{t}^{O} \neq \emptyset, \forall t \geq 0\right\}=\left\{\beta_{n} \neq \emptyset \text { for all } n \geq 0\right\}
$$

The proof of Equation (2.4) relies heavily on Equation (5.1) and the following two lemmas.

Lemma 5.1. Let $\left\{W_{n}\right\}_{n \geq 0}$ be the branching process with random vertex weights defined as in Section 3 and $\sigma_{0} \in\left(0, \frac{1}{10 \log \left(\lambda M^{2}\right)}\right)$ defined as in Section 4 , then

$$
\liminf _{d \rightarrow+\infty} \widehat{P}_{\lambda, d}\left(W_{\left\lfloor\sigma_{0} \log d\right\rfloor}=\emptyset\right) \geq E\left(\frac{1}{1+\lambda \rho \theta}\right),
$$

where $\widehat{P}_{\lambda, d}$ is the annealed measure of the branching process defined as in Section 3.

Lemma 5.2. Let $\left\{V_{n}\right\}_{n \geq 0}$ be defined as in Section 4, then

$$
\lim _{d \rightarrow+\infty}\left[P_{\lambda, d}\left(\beta_{\left\lfloor\sigma_{0} \log d\right\rfloor}=\emptyset\right)-P_{\lambda, d}\left(V_{\left\lfloor\sigma_{0} \log d\right\rfloor}=\emptyset\right)\right]=0 .
$$

The proof of Lemma 5.1 is given in Subsection 5.1. The core idea of the proof is to show that the branching process survives with high probability conditioned on $W_{\left\lfloor\sigma_{0} \log d\right\rfloor} \neq \emptyset$. The proof of Lemma 5.2 is given in Subsection 5.2. The core idea of the proof is to construct a coupling of $\left\{\beta_{n}\right\}_{n \geq 0}$ and $\left\{V_{n}\right\}_{n \geq 0}$ such that $\beta_{\left\lfloor\sigma_{0} \log d\right\rfloor}=$ $V_{\left\lfloor\sigma_{0} \log d\right\rfloor}$ with high probability. Now we show how to utilize Lemmas 5.1 and 5.2 to prove Equation (2.4).

Proof of Equation (2.4): We couple $\left\{W_{n}\right\}_{n \geq 0}$ and $\left\{V_{n}\right\}_{n \geq 0}$ under the same probability space as what we have done in Subsection 4.1. Recalling that we define $B(d)$ as the event that the coupling is successful at step $m$ for all $m \leq\left\lfloor\sigma_{0} \log d\right\rfloor$, then

$$
V_{\left\lfloor\sigma_{0} \log d\right\rfloor}=W_{\left\lfloor\sigma_{0} \log d\right\rfloor}
$$

on the event $B(d)$. Therefore, by Lemma 4.3,

$$
\left|\widehat{P}_{\lambda, d}\left(W_{\left\lfloor\sigma_{0} \log d\right\rfloor}=\emptyset\right)-P_{\lambda, d}\left(V_{\left\lfloor\sigma_{0} \log d\right\rfloor}=\emptyset\right)\right| \leq 2 P_{\lambda, d}\left(B(d)^{c}\right) \rightarrow 0
$$

as $d \rightarrow+\infty$ and hence

$$
\liminf _{d \rightarrow+\infty} P_{\lambda, d}\left(V_{\left\lfloor\sigma_{0} \log d\right\rfloor}=\emptyset\right) \geq E\left(\frac{1}{1+\lambda \rho \theta}\right)
$$

according to Lemma 5.1. Then, by Lemma 5.2,

$$
\liminf _{d \rightarrow+\infty} P_{\lambda, d}\left(\beta_{\left\lfloor\sigma_{0} \log d\right\rfloor}=\emptyset\right) \geq E\left(\frac{1}{1+\lambda \rho \theta}\right)
$$

and hence

$$
\liminf _{d \rightarrow+\infty} P_{\lambda, d}\left(\beta_{n}=\emptyset \text { for some } n \geq 0\right) \geq E\left(\frac{1}{1+\lambda \rho \theta}\right) .
$$

By Equation (5.2),

$$
\limsup _{d \rightarrow+\infty} P_{\lambda, d}\left(\beta_{n} \neq \emptyset \text { for all } n \geq 0\right) \leq E\left(\frac{\lambda \rho \theta}{1+\lambda \rho \theta}\right) .
$$

Equation (2.4) follows from Equations (5.1) and (5.3) directly. 
5.1. Proof of Lemma 5.1. In this subsection, we give the proof of Lemma 5.1. Let $\left\{\widehat{W}_{n}\right\}_{n \geq 0}$ be defined as in Subsection 4.2 , then $\left\{\widehat{W}_{n}\right\}_{n \geq 0}$ is a branching process with random vertex weights on a subtree of $\mathbb{T}^{d}$ which is isomorphic to $\mathbb{T}^{d-\left\lfloor\frac{d}{N(d)}\right\rfloor}$ as we have introduced. For each $n \geq 0, \widehat{W}_{n} \subseteq W_{n}$. The following lemma is crucial for us to prove Lemma 5.1.

Lemma 5.3. For any $\sigma \in\left(0, \frac{1}{10 \log \left(\lambda M^{2}\right)}\right)$,

$$
\lim _{d \rightarrow+\infty} \widehat{P}_{\lambda, d}\left(W_{n} \neq \emptyset \text { for all } n \geq 0 \mid \widehat{W}_{\lfloor\sigma \log d\rfloor-1} \neq \emptyset\right)=1
$$

We give the proof of Lemma 5.3 at the end of this subsection. Now we show how to utilize Lemma 5.3 to prove Lemma 5.1.

Proof of Lemma 5.1: By the conditional probability formula,

$$
\begin{aligned}
& \widehat{P}_{\lambda, d}\left(W_{n} \neq \emptyset \text { for all } n \geq 0\right) \\
& \geq \widehat{P}_{\lambda, d}\left(\left(W_{n} \neq \emptyset \text { for all } n \geq 0 \mid \widehat{W}_{\lfloor\sigma \log d\rfloor-1} \neq \emptyset\right) \widehat{P}_{\lambda, d}\left(\widehat{W}_{\lfloor\sigma \log d\rfloor-1} \neq \emptyset\right) .\right.
\end{aligned}
$$

Then, by Lemmas 3.1 and 5.3,

$$
\limsup _{d \rightarrow+\infty} \widehat{P}_{\lambda, d}\left(\widehat{W}_{\lfloor\sigma \log d\rfloor-1} \neq \emptyset\right) \leq E\left(\frac{\lambda \rho \theta}{1+\lambda \rho \theta}\right)
$$

and hence

$$
\liminf _{d \rightarrow+\infty} \widehat{P}_{\lambda, d}\left(\widehat{W}_{\lfloor\sigma \log d\rfloor}=\emptyset\right) \geq \liminf _{d \rightarrow+\infty} \widehat{P}_{\lambda, d}\left(\widehat{W}_{\lfloor\sigma \log d\rfloor-1}=\emptyset\right) \geq E\left(\frac{1}{1+\lambda \rho \theta}\right)
$$

for any $\sigma \in\left(0, \frac{1}{10 \log \left(\lambda M^{2}\right)}\right)$.

For given $\lambda>\frac{1}{E\left(\rho^{2}\right)}$ and $\sigma_{0} \in\left(0, \frac{1}{10 \log \left(\lambda M^{2}\right)}\right)$, we choose arbitrary $\hat{\lambda} \in(\lambda,+\infty)$ and $\sigma \in\left(0, \sigma_{0}\right)$. For sufficiently large $d$, we define

$$
\widehat{d}=\inf \left\{k: k-\left\lfloor\frac{k}{N(k)}\right\rfloor \geq d\right\},
$$

then it is easy to check that $\lim _{d \rightarrow+\infty} \frac{\widehat{d}}{d}=1$ and hence

$$
\frac{\widehat{\lambda}}{\widehat{d}} \geq \frac{\lambda}{d} \text { while } \sigma \log \widehat{d} \leq \sigma_{0} \log d
$$

for sufficiently large $d$. As we have introduced, $\left\{\widehat{W}_{n}\right\}_{n \geq 0}$ on $\mathbb{T}^{\widehat{d}}$ can be identified with $\left\{W_{n}\right\}_{n \geq 0}$ on $\mathbb{T}^{\widehat{d}-\left\lfloor\frac{\widehat{d}}{N(\widehat{d})}\right\rfloor}$ with a scaling of the infection rate $\lambda$. As a result, by Equation (5.5),

$$
\widehat{P}_{\widehat{\lambda}, \widehat{d}}\left(\widehat{W}_{\lfloor\sigma \log \widehat{d}\rfloor}=\emptyset\right) \leq \widehat{P}_{\lambda, d}\left(W_{\left\lfloor\sigma_{0} \log d\right\rfloor}=\emptyset\right)
$$

for sufficiently large $d$. By Equations (5.4) and (5.6),

$$
\liminf _{d \rightarrow+\infty} \widehat{P}_{\lambda, d}\left(W_{\left\lfloor\sigma_{0} \log d\right\rfloor}=\emptyset\right) \geq \liminf _{d \rightarrow+\infty} \widehat{P}_{\widehat{\lambda}, \widehat{d}}\left(\widehat{W}_{\lfloor\sigma \log \widehat{d}\rfloor}=\emptyset\right) \geq E\left(\frac{1}{1+\widehat{\lambda} \rho \widehat{\theta}}\right),
$$

where $\widehat{\theta}$ satisfies

$$
E\left(\frac{\widehat{\lambda} \rho^{2}}{1+\widehat{\lambda} \rho \widehat{\theta}}\right)=1
$$

and it is easy to check that $\lim _{\widehat{\lambda} \rightarrow \lambda} \widehat{\theta}=\theta$. 
Let $\widehat{\lambda} \rightarrow \lambda$, then Lemma 5.1 follows directly from Equation (5.7).

At the end of this subsection we give the proof of Lemma 5.3.

Proof of Lemma 5.3: Let

$$
\begin{aligned}
& \widehat{D}_{\sigma}=\left\{y: \text { there exists } x \in \bigcup_{m=0}^{\lfloor\sigma \log d\rfloor-1} \widehat{W}_{m}\right. \\
& \text { such that } y=x(i) \text { for some } i \geq j(d) \text { and } U(x, y)<Y(x)\},
\end{aligned}
$$

i.e, $\widehat{D}$ defined in Subsection 4.2 equals $\widehat{D}_{\sigma_{0}}$. According to Lemma 4.4,

$$
\lim _{d \rightarrow+\infty} \widehat{P}_{\lambda, d}\left(\left|\widehat{D}_{\sigma}\right| \geq\left\lfloor\frac{\sqrt{\log d}}{N(d)}\right\rfloor \mid \widehat{W}_{\lfloor\sigma \log d\rfloor-1} \neq \emptyset\right)=1 .
$$

Note that although $\sigma_{0}$ is fixed in Lemma 4.4, the analysis leading to Lemma 4.4 holds for any $\sigma<\frac{1}{10 \log \left(\lambda M^{2}\right)}$. According to Equation (4.17),

$$
\begin{aligned}
& \lim _{d \rightarrow+\infty} \widehat{P}_{\lambda, d}\left(\sum_{u \in \widehat{D}_{\sigma}} \chi(u) \geq\left\lfloor\frac{\lambda \epsilon \theta}{2(1+\lambda \epsilon \theta)} \frac{\sqrt{\log d}}{N(d)}\right\rfloor \mid \widehat{W}_{\lfloor\sigma \log d\rfloor-1} \neq \emptyset\right. \\
&\left.\qquad\left|\widehat{D}_{\sigma}\right| \geq\left\lfloor\frac{\log d}{N(d)}\right\rfloor\right)=1 .
\end{aligned}
$$

By Equations (5.8), (5.9) and the conditional probability formula,

$$
\lim _{d \rightarrow+\infty} \widehat{P}_{\lambda, d}\left(\sum_{u \in \widehat{D}_{\sigma}} \chi(u) \geq\left\lfloor\frac{\lambda \epsilon \theta}{2(1+\lambda \epsilon \theta)} \frac{\sqrt{\log d}}{N(d)}\right\rfloor \mid \widehat{W}_{\lfloor\sigma \log d\rfloor-1} \neq \emptyset\right)=1 .
$$

If $\chi(u)=1$ for some $u \in \widehat{D}_{\sigma}$, then infinitely many vertices have ever been infected. Therefore,

$$
\left\{\sum_{u \in \widehat{D}_{\sigma}} \chi(u) \geq\left\lfloor\frac{\lambda \epsilon \theta}{2(1+\lambda \epsilon \theta)} \frac{\sqrt{\log d}}{N(d)}\right\rfloor\right\} \subseteq\left\{W_{n} \neq \emptyset \text { for all } n \geq 0\right\} .
$$

As a result, Lemma 5.3 follows from Equation (5.10) directly.

5.2. Proof of Lemma 5.2. In this subsection we give the proof of Lemma 5.2. First we couple $\left\{\beta_{n}\right\}_{n \geq 0}$ and $\left\{V_{n}\right\}_{n \geq 0}$ under the same probability space. Let $\{\widetilde{Y}(x)\}_{x \in \mathbb{Z}_{+}^{d}}$ and $\{\widetilde{U}(x, y)\}_{x \in \mathbb{Z}_{+}^{d}, x \rightarrow y}$ be defined as in Section 4 , then $\left\{V_{n}\right\}_{n \geq 0}$ is defined as in Section 4 according to the values of $\{\widetilde{Y}(x)\}_{x \in \mathbb{Z}_{+}^{d}}$ and $\{\widetilde{U}(x, y)\}_{x \in \mathbb{Z}_{+}^{d}, x \rightarrow y}$. For any $x, y \in \mathbb{Z}_{+}^{d}, x \rightarrow y$, let $\widetilde{U}_{2}(x, y)$ be an independent copy of $\widetilde{U}(x, y)$ under the quenched measure. We assume that all these exponential times are independent under the quenched measure. For the contact process, we let $\tilde{Y}(x)$ be the time $x$ waits for to become healthy after the first moment when $x$ is infected. We let $\widetilde{U}(x, y)$ be the time $x$ waits for to infect $y$ after the first moment when $x$ is infected. If $\widetilde{U}(x, y)<\widetilde{Y}(x)$, then after the first infection from $x$ to $y, x$ waits for $\widetilde{U}_{2}(x, y)$ units of time to infect $y$ again, i.e., $x$ infects $y$ at least twice before becoming healthy when $\widetilde{U}(x, y)+\widetilde{U}_{2}(x, y)<\widetilde{Y}(x)$. Following the above definitions, $\left\{V_{n}\right\}_{n \geq 0}$ and $\left\{\beta_{n}\right\}_{n \geq 0}$ 
are coupled under the same probability space and it is obvious that $V_{n} \subseteq \beta_{n}$ for each $n \geq 0$.

Let $\bar{J}(d)$ be defined as in Section 4, i.e., the event that $\widetilde{U}(x, y)>\widetilde{Y}(x)$ and $\widetilde{U}(z, y)>\widetilde{Y}(z)$ for any $x, y, z$ that $x, z \in \bigcup_{m=0}^{\left\lfloor\sigma_{0} \log d\right\rfloor-1} V_{m}$ and $x, z \rightarrow y$. On the event $J(d)$, if $V_{\left\lfloor\sigma_{0} \log d\right\rfloor} \neq \beta_{\left\lfloor\sigma_{0} \log d\right\rfloor}$, then there must exist repeated infection from some $x$ to $y$ that $x \rightarrow y$ for the contact process, i.e.,

$$
\widetilde{U}(x, y)+\widetilde{U}_{2}(x, y)<\tilde{Y}(x) .
$$

For each $m \geq 1, L_{m}(O)$ is the set of oriented paths on $\mathbb{Z}_{+}^{d}$ starting at $O$ with length $m$ defined as in Section 4. For each $\vec{l}: O=l_{0} \rightarrow l_{1} \rightarrow l_{2} \rightarrow \ldots \rightarrow l_{m}$ in $L_{m}(O)$, we denote by $\widehat{A}_{\vec{l}}$ the event that $\widetilde{U}\left(l_{i}, l_{i+1}\right)<\widetilde{Y}\left(l_{i}\right)$ for all $0 \leq i \leq m-2$ and

$$
\widetilde{U}\left(l_{m}-1, l_{m}\right)+\widetilde{U}_{2}\left(l_{m-1}, l_{m}\right)<\tilde{Y}\left(l_{m-1}\right)
$$

then according to the aforesaid analysis,

$$
P_{\lambda, d}\left(V_{\left\lfloor\sigma_{0} \log d\right\rfloor} \neq \beta_{\left\lfloor\sigma_{0} \log d\right\rfloor}, J(d)\right) \leq \sum_{m=0}^{\left\lfloor\sigma_{0} \log d\right\rfloor} \sum_{\vec{l} \in L_{m}} P_{\lambda, d}\left(\widehat{A}_{\vec{l}}\right) .
$$

Now we give the proof of Lemma 5.2.

Proof of Lemma 5.2: For each $\vec{l} \in L_{m}$, since $\widetilde{U}(\cdot, \cdot), \widetilde{U}_{2}(\cdot, \cdot)$ are exponential times with rate at most $\frac{\lambda M^{2}}{d}$ while $\tilde{Y}(\cdot)$ is an exponential time with rate one, it is easy to check that

$$
P_{\lambda, d}\left(\widehat{A}_{\vec{l}}\right) \leq\left(\frac{\lambda M^{2}}{d}\right)^{m-1}\left(\frac{\lambda M^{2}}{d}\right)^{2}=\frac{\lambda^{m+1} M^{2 m+2}}{d^{m+1}} .
$$

Since $\left|L_{m}\right|=d^{m}$ and $\sigma_{0}<\frac{1}{10 \log \left(\lambda M^{2}\right)}$, by Equation (5.11),

$$
P_{\lambda, d}\left(V_{\left\lfloor\sigma_{0} \log d\right\rfloor} \neq \beta_{\left\lfloor\sigma_{0} \log d\right\rfloor}, J(d)\right) \leq \sum_{m=0}^{\left\lfloor\sigma_{0} \log d\right\rfloor} d^{m} \frac{\lambda^{m+1} M^{2 m+2}}{d^{m+1}} \leq \frac{\lambda^{2} M^{4} d^{-0.9}}{\lambda M^{2}-1} .
$$

By Equation (5.12),

$$
\begin{aligned}
& \left|P_{\lambda, d}\left(\beta_{\left\lfloor\sigma_{0} \log d\right\rfloor}=\emptyset\right)-P_{\lambda, d}\left(V_{\left\lfloor\sigma_{0} \log d\right\rfloor}=\emptyset\right)\right| \\
& \leq 2 P_{\lambda, d}\left(\beta_{\left\lfloor\sigma_{0} \log d\right\rfloor} \neq V_{\left\lfloor\sigma_{0} \log d\right\rfloor}\right) \\
& \leq 2 P_{\lambda, d}\left(\beta_{\left\lfloor\sigma_{0} \log d\right\rfloor} \neq V_{\left\lfloor\sigma_{0} \log d\right\rfloor}, J(d)\right)+2 P_{\lambda, d}\left(J(d)^{c}\right) \\
& \leq \frac{2 \lambda^{2} M^{4} d^{-0.9}}{\lambda M^{2}-1}+2 P_{\lambda, d}\left(J(d)^{c}\right) .
\end{aligned}
$$

We claim that

$$
\lim _{d \rightarrow+\infty} P_{\lambda, d}(J(d))=1 .
$$

Equation (5.14) follows from the following analysis, which we have utilized in the proof of Lemma 4.3. For each $0 \leq m \leq\left\lfloor\sigma_{0} \log d\right\rfloor-1$, according to the fact that $\widetilde{Y}(\cdot)$ is an exponential time with rate 1 while $\widetilde{U}(\cdot, \cdot)$ is an exponential time with rate at most $\frac{\lambda}{d} M^{2}$ and $|q(x)| \leq\left|V_{m}\right|$ for any $x \in V_{m}$,

$$
P_{\lambda, d}\left(J(d, m) \mid V_{m} \leq d^{0.2}\right) \geq 1-d^{0.2} \times d^{0.2} \frac{\lambda}{d} M^{2}=1-\lambda M^{2} d^{-0.6} .
$$


Then, by Equation (4.12),

$$
P_{\lambda, d}(J(d, m)) \geq\left(1-\lambda M^{2} d^{-0.6}\right)\left(1-\frac{d^{-0.1}}{\lambda M^{2}-1}\right)
$$

for all $0 \leq m \leq\left\lfloor\sigma_{0} \log d\right\rfloor-1$ and hence

$$
P_{\lambda, d}(J(d)) \geq 1-\left\lfloor\sigma_{0} \log d\right\rfloor\left[1-\left(1-\lambda M^{2} d^{-0.6}\right)\left(1-\frac{d^{-0.1}}{\lambda M^{2}-1}\right)\right],
$$

and Equation (5.14) follows from which directly.

Lemma 5.2 follows directly from Equations (5.13) and (5.14).

\section{Acknowledgements}

We are grateful to the reviewers. Their comments are great help for us to improve this paper. We are grateful to the financial support from Beijing Jiaotong University with grant number 2016RC034 and the financial support from the National Natural Science Foundation of China with grant number 11501542.

\section{References}

D. Bertacchi, N. Lanchier and F. Zucca. Contact and voter processes on the infinite percolation cluster as models of host-symbiont interactions. Ann. Appl. Probab. 21 (4), 1215-1252 (2011). MR2857447.

J. T. Cox and R. Durrett. Oriented percolation in dimensions $d \geq 4$ : bounds and asymptotic formulas. Math. Proc. Cambridge Philos. Soc. 93 (1), 151-162 (1983). MR684285.

T. E. Harris. Contact interactions on a lattice. Ann. Probability 2, 969-988 (1974). MR0356292.

T. M. Liggett. Interacting particle systems, volume 276 of Grundlehren der Mathematischen Wissenschaften [Fundamental Principles of Mathematical Sciences]. Springer-Verlag, New York (1985). ISBN 0-387-96069-4. MR776231.

T. M. Liggett. Stochastic interacting systems: contact, voter and exclusion processes, volume 324 of Grundlehren der Mathematischen Wissenschaften [Fundamental Principles of Mathematical Sciences]. Springer-Verlag, Berlin (1999). ISBN 3-540-65995-1. MR1717346.

Y. Pan, D. Chen and X. Xue. Contact process on regular tree with random vertex weights. Front. Math. China 12 (5), 1163-1181 (2017). ISSN 1673-3452. MR3698419.

J. Peterson. The contact process on the complete graph with random vertexdependent infection rates. Stochastic Process. Appl. 121 (3), 609-629 (2011). MR2763098.

R. H. Schonmann and M. E. Vares. The survival of the large-dimensional basic contact process. Probab. Theory Relat. Fields 72 (3), 387-393 (1986). MR843501.

$\mathrm{X}$. Xue. Contact processes with random vertex weights on oriented lattices. ALEA Lat. Am. J. Probab. Math. Stat. 12 (1), 245-259 (2015). MR3343484.

X. Xue. Mean field limit for survival probability of the high-dimensional contact process. Statist. Probab. Lett. 127, 178-184 (2017a). MR3648309.

X. Xue. Survival probabilities of high-dimensional stochastic SIS and SIR models with random edge weights. ArXiv Mathematics e-prints (2017b). arXiv: 1706.08233. 
Q. Yao and X. Chen. The complete convergence theorem holds for contact processes in a random environment on $\mathbb{Z}^{d} \times \mathbb{Z}^{+}$. Stochastic Process. Appl. 122 (9), 30663100 (2012). MR2946436. 